\title{
12. MINERALOGY OF MARK PERIDOTITES: REPLACEMENT THROUGH MAGMA CHANNELING EXAMINED FROM HOLE 920D, MARK AREA ${ }^{1}$
}

\author{
Kiyoaki Niida ${ }^{2}$
}

\begin{abstract}
Peridotites drilled at Site 920 of Ocean Drilling Program Leg 153 in the Mid-Atlantic Ridge near the Kane Transform (MARK) area were examined to understand the mode of magma channeling and modification of the upper mantle peridotites This paper presents mineralogical data from a slow-spreading ocean ridge for peridotites that have been modified by magma channeling and for those that are free from the modification.

The chemical compositions of primary minerals in spinel harzburgite and lherzolite are highly uniform. Olivine core compositions concentrate around $\mathrm{Fo}=91.0$ and $\mathrm{NiO}=0.37 \mathrm{wt} \%$. Spinel cores also have a small compositional range of $\mathrm{Mg} \#=70$ 75 and $\mathrm{Cr} \#=27-31$. Orthopyroxenes with $\mathrm{Mg} \#=90.5-91.6$ have $\mathrm{Al}_{2} \mathrm{O}_{3}$ contents of 3.17 to $4.77 \mathrm{wt} \%$. $\mathrm{TiO}_{2}$ and $\mathrm{Na}_{2} \mathrm{O}$ contents in primary clinopyroxenes are extremely low, less than 0.15 and $0.11 \mathrm{wt} \%$, respectively. These results suggest that the MARK peridotites represent moderately depleted upper mantle peridotite. The chemical compositions of primary minerals are modified where the peridotites are crosscut by gabbro and pyroxenite dikes and veins. Such peridotites are enriched in $\mathrm{FeO}^{*}$ and $\mathrm{TiO}_{2}$, and depleted in $\mathrm{MgO}, \mathrm{NiO}$, and $\mathrm{Al}_{2} \mathrm{O}_{3}$.

Spatial variations in chemical composition of minerals were examined across channels in Samples 153-920D-10R-2 (Piece $4,35-41 \mathrm{~cm}$ ) and 153-920D-13R-2 (Piece 7, 136-143 cm). Primary minerals in spinel harzburgite are enriched in FeO* and $\mathrm{TiO}_{2}$ within narrow replacement bands next to websterite veins. No evidence for melt extractions was detected in the harzburgite wall, indicating that the replacement occurred as a one-way modification process by melt addition from the channeling magma. The Fo-NiO systematics for the modified olivines support a model of open-system reaction between the wall harzburgite and the infiltration melt with a highly evolved composition. All of the results emphasize that the final magma channeling occurred in "cold" peridotites at a shallow level of the upper mantle.
\end{abstract}

\section{INTRODUCTION}

Most abyssal peridotites have been dredged from oceanic fracture zones because they only rarely are exposed along mid-ocean ridges, and have been used as representatives for suboceanic peridotites (e.g., Bonatti and Honnorez, 1976; Dick and Bullen, 1984; Dick, 1989; Bonatti and Michael, 1989). Ocean Drilling Program (ODP) Leg 153 drilled at Site 920 in the Mid-Atlantic Ridge near the Kane Transform (MARK) area to achieve deep penetration into peridotites. These peridotites are believed to be representative of the upper mantle created at a slow-spreading ridge. Hole $920 \mathrm{~B}$ penetrated peridotites to 126.4 meters below seafloor (mbsf) with a total cumulative core recovery of $47.78 \mathrm{~m}(39.7 \%)$. Hole 920D penetrated to 200.8 mbsf with a core recovery of $95.08 \mathrm{~m}(47.4 \%)$. Both Holes $920 \mathrm{~B}$ and $920 \mathrm{D}$ were historic in terms of the length of penetration in oceanic peridotites and the core recovery.

Ocean-ridge peridotites appear to be pervasively modified by channeling magma in the upper mantle, as discussed for ophiolitic peridotites (e.g., Dick, 1977; Quick, 1981; Nicolas and Prinzhofer, 1983; Boudier and Nicolas, 1995), orogenic lherzolites (e.g., Bodinier et al., 1990; Takazawa et al., 1992), and subcontinental xenoliths (e.g., Frey and Green, 1974; Wilshire and Shervais, 1975; Irving, 1980; Kempton, 1987; Nielson et al., 1993). Recent reports on impregnated peridotites dredged from the East Pacific Rise (Cannat et al., 1990; Hekinian et al., 1993; Girardeau and Francheteau, 1993) and drilled peridotites from Site 895 of ODP Leg 147 to Hess Deep (Arai and Matsukage, 1996) suggest the role of melt per-

'Karson, J.A., Cannat, M., Miller, D.J., and Elthon, D. (Eds.), 1997. Proc, ODP, Sci. Results, 153: College Station, TX (Ocean Drilling Program).

${ }^{2}$ Department of Earth and Planetary Sciences, Graduate School of Science, Hokkaido University, Sapporo, 060 Japan. kiyo@epms.hokudai.ac.jp colation as a pervasive process of magma channeling in very shallow mantle peridotites.

Peridotites drilled at Site 920 in the MARK area were examined to understand the mode of magma channeling in the upper mantle beneath the Mid-Atlantic Ridge and how the wall peridotites are modified by magma channeling. This paper presents mineralogical data for suboceanic-ridge peridotites that have been modified by magma channeling and those that are free from such modifications. The results emphasize that the final magma channeling occurred in cold mantle peridotites at a shallow level.

\section{SAMPLE LOCATIONS}

Serpentinized peridotite crops out on the western wall of the axial valley of the Mid-Atlantic Ridge near $23^{\circ} \mathrm{N}$, close to the Kane Fracture zone. Site $920\left(23^{\circ} 20^{\prime} \mathrm{N}\right)$ is located in the northern portion of the serpentinite exposure, which can be traced more than $20 \mathrm{~km}$ along the valley wall from $3100 \mathrm{mbsf}$ to $3500 \mathrm{mbsf}$ (see fig. 3 in Cannat, Karson, Miller, et al., 1995) and has been surveyed during dives of the submersibles Alvin (Karson et al., 1987) and Nautile (Mével et al., 1991). Serpentinized peridotite was drilled at the southern extension of the exposure at Site $670\left(23^{\circ} 10^{\prime} \mathrm{N}\right)$ during ODP Leg 109 (Fujii, 1990; Hébert et al., 1990; Juteau et al., 1990; Komor et al., 1990).

\section{PETROGRAPHY}

All the peridotite samples examined in this paper are from Hole $920 \mathrm{D}$, which yielded rocks that are lithologically similar to those in Hole 920B. Cores recovered from Hole 920D are 95\% serpentinized harzburgite with small amounts of serpentinized lherzolite and dun- 
ite. Primary minerals are olivine, orthopyroxene, clinopyroxene, and spinel. Modal proportion of total pyroxenes (orthopyroxene + clinopyroxene) varies from $0 \%$ to $40 \%$ in the Hole $920 \mathrm{D}$ peridotites. In rare cases, pyroxene-rich harzburgite gradually becomes lherzolitic, containing more than $5 \%$ clinopyroxenes. The dunite is subdivided into two types: orthopyroxene-free spinel dunite and orthopyroxenebearing spinel dunite. These peridotites are moderately to pervasively serpentinized $(50 \%-100 \%$ altered).

The extensive peridotite sections recovered in the cores from Hole $920 \mathrm{D}$ are characterized by numerous plagioclase and/or clinopyroxene-bearing plutonic and hypabyssal dikes, veins, and veinlets, crosscutting the peridotites. Such magmatic intrusive rocks include olivine gabbro, gabbro, oxide-rich gabbro, pyroxenite, oxide-rich pyroxenite, and plagioclase-olivine phyric diabase. The maximum width of diabase dikes exceeds $3 \mathrm{~m}$ in total length of cores recovered from of Hole 920D (Unit 6), but the gabbro and pyroxenite dikes are generally less than $35 \mathrm{~cm}$ wide. A total of 138 centimeter-to millimeter-thick magmatic veins and veinlets were found in the cores from Hole 920D (Cannat, Karson, Miller, et al., 1995).

\section{Dunite}

Orthopyroxene-bearing spinel dunites are commonly observed in close association with magmatic intrusive rocks. In Section 153920D-11R-1, orthopyroxene-bearing dunite bands, $30 \mathrm{~cm}$ thick, are in contact with a 25 -cm-thick gabbro dike crosscutting the spinel harzburgite wall. The contact between the dunite and harzburgite was recovered in Piece $2(11-19 \mathrm{~cm})$ of the section. The dunite portion of the piece consists of $96 \%$ olivine, $2 \%$ orthopyroxene, and $2 \%$ spinel, whereas the harzburgite portion consists of $84 \%$ olivine, $14 \%$ orthopyroxene, $1 \%$ clinopyroxene, and $1 \%$ spinel (Table 1 ). Modal depletion of pyroxenes in harzburgite can be traced in this section toward the contact of gabbro dike through the dunite.

Another dunite examined here (Sample 153-920D-2R-1, Piece 4, $21-24 \mathrm{~cm}$ ) is composed of $94 \%$ olivine, $5 \%$ orthopyroxene, and $1 \%$ spinel, and is in contact with the piece crosscut by a gabbro vein. Grain size of orthopyroxenes $(<2.5 \mathrm{~mm})$ and spinels $(<0.5 \mathrm{~mm})$ are definitely smaller than those in the wall harzburgite. The spinel grains generally display euhedral to subhedral shape in dunites, indicating a magmatic crystallization.

\section{Harzburgite and Lherzolite}

Spinel peridotites examined contain 66-84 modal\% olivine, $14 \%-28 \%$ orthopyroxene, $1 \%-6 \%$ clinopyroxene, and $1 \%-2 \%$ spinel, as listed in Table 1. Modal abundances of orthopyroxenes and clinopyroxenes are variable gradationally in the peridotite sections in Hole 920D. In rare cases of Samples 153-920D-22R-2 (Piece 1A, 4$9 \mathrm{~cm}$ ) and 153-920D-22R-2 (Piece 1D, 73-77 cm), the clinopyroxene abundance attains more than $5 \%$ of the mode, which qualifies the peridotites as lherzolites, based on the systematics of igneous rocks by the IUGS subcommission (Streckeisen, 1973). Primary mineral assemblages in the spinel harzburgites and lherzolites are characterized by porphyroclastic textures consisting of large porphyroclastic grains of orthopyroxene and olivine with a highly recrystallized finegrained matrix. The maximum size of porphyroclastic grains rarely exceeds $1 \mathrm{~cm}$. In general, however, clinopyroxene grains do not show any porphyroclastic features. They commonly appear as interstitial grains among strained olivine and orthopyroxene grains and/or in monomineralic clinopyroxene aggregates, and sometimes polymineralic aggregates with orthopyroxenes and spinels. "Clinopyroxene trains," defined by interconnected or discontinuous chains of clinopyroxenes, are commonly observed in peridotite sections from both Holes 920B and 920D. Magmatically twinned clinopyroxenes have been reported from Sections 153-920B-3R-2 and 920D-15R-4 (Cannat, Karson, Miller, et al., 1995). Spinel trains are more commonly observed in peridotite sections. No clear connection of such magmatic features to any gabbroic or pyroxenitic veins has been described in the peridotite sections.

\section{CHEMICAL COMPOSITION OF PRIMARY MINERALS}

Primary constituent minerals in the MARK peridotites were analyzed using the JEOL JCMA-733 electron probe microanalyzer at Hokkaido University. Operating conditions were $15 \mathrm{kV}$ and $0.02 \mathrm{~mA}$ probe current, and standard ZAF data-reduction procedures were employed. Representative analyses for olivine, spinel, orthopyroxene, and clinopyroxene are listed in Tables 2 to 5 ; these analyses are averages of 3-37 point analyses on the cores of primary minerals.

\section{Olivine}

Olivine is the most abundant phase in the MARK peridotites. The forsterite (Fo) content of olivine cores in the spinel harzburgites and lherzolites ranges from 90.3 to 91.5 , indicating the moderately depleted nature of the upper mantle peridotites (Table 2). The NiO contents of olivine cores vary from 0.27 to $0.44 \mathrm{wt} \%$, which corresponds to the compositional range of mantle olivines (Takahashi et al., 1987). The olivines are notably uniform in composition. As shown in Figure 1, olivine core compositions cluster around $\mathrm{Fo}=91.0$ and $\mathrm{NiO}$

Table 1. Petrographic summary of peridotites examined from Hole 920D.

\begin{tabular}{|c|c|c|c|c|c|c|c|}
\hline $\begin{array}{l}\text { Core, section, } \\
\text { interval }(\mathrm{cm})\end{array}$ & $\begin{array}{r}\text { Depth } \\
\text { (mbsf) }\end{array}$ & $\begin{array}{l}\text { Olivine } \\
(\%)\end{array}$ & $\begin{array}{l}\text { Orthopyroxene } \\
\text { (\%) }\end{array}$ & $\begin{array}{c}\text { Clinopyroxene } \\
(\%)\end{array}$ & $\begin{array}{l}\text { Spinel } \\
(\%)\end{array}$ & Rock type & Remarks \\
\hline \multicolumn{8}{|c|}{ Orthopyroxene-bearing spinel dunite: } \\
\hline $\begin{array}{l}\text { 153-920D- } \\
\text { 2R-1 (Piece 4, 21-24) } \\
11 \mathrm{R}-1 \text { (Piece 2, 15-19) }\end{array}$ & $\begin{array}{r}8.02 \\
85.41\end{array}$ & $\begin{array}{l}94 \\
96\end{array}$ & $\begin{array}{l}5 \\
2\end{array}$ & $\begin{array}{l}0 \\
0\end{array}$ & $\begin{array}{l}1 \\
2\end{array}$ & $\begin{array}{l}\text { opx-sp.D } \\
\text { opx-sp.D }\end{array}$ & $\begin{array}{l}\text { Crosscut by gabbro vein in Piece } 5 \text {. } \\
\text { In contact with gabbro (Unit } 8 \text { ). }\end{array}$ \\
\hline \multicolumn{8}{|l|}{ Spinel harzburgite-Iherzolite: } \\
\hline $\begin{array}{l}\text { 153-920D- } \\
\text { 11R-1 (Piece 2, 11-15) } \\
\text { 14R-3 (Piece 1, 29-34) } \\
\text { 22R-2 (Piece 1A, 4-9) } \\
\text { 22R-2 (Piece 1D, 73-77) } \\
\text { 22R-3 (Piece 1A, 38-43) } \\
\text { 22R-4 (Piece 4, 104-109) } \\
\text { 22R-5 (Piece 3, 75-80) } \\
\text { 22R-5 (Piece 6C, 120-128) } \\
\text { 22R-6 (Piece 12, 111-117) } \\
\text { 22R-7 (Piece 4, 56-61) }\end{array}$ & $\begin{array}{r}85.41 \\
117.32 \\
191.84 \\
192.53 \\
193.11 \\
194.47 \\
195.38 \\
195.83 \\
197.15 \\
197.89\end{array}$ & $\begin{array}{l}84 \\
75 \\
73 \\
68 \\
75 \\
74 \\
66 \\
78 \\
75 \\
73\end{array}$ & $\begin{array}{l}14 \\
20 \\
21 \\
24 \\
19 \\
22 \\
28 \\
17 \\
20 \\
22\end{array}$ & $\begin{array}{l}1 \\
4 \\
5 \\
6 \\
4 \\
3 \\
4 \\
4 \\
4 \\
4\end{array}$ & $\begin{array}{l}1 \\
1 \\
1 \\
2 \\
2 \\
1 \\
2 \\
1 \\
1 \\
1\end{array}$ & $\begin{array}{l}\text { sp. H } \\
\text { sp. H } \\
\text { sp. L } \\
\text { sp. L } \\
\text { sp. H } \\
\text { sp. H } \\
\text { sp. H } \\
\text { sp. H } \\
\text { sp. H } \\
\text { sp. H }\end{array}$ & $\begin{array}{l}\text { In contact with oxide gabbro (Pieces 7-8), } \\
\text { Crosscut by gabbro vein in Pieces 6-9. }\end{array}$ \\
\hline
\end{tabular}

Notes: Point-counting modal estimates $(\%)$ for minerals: ol =olivine; opx = orthopyroxene; $\mathrm{cpx}=$ clinopyroxene; $\mathrm{sp}=\mathrm{spinel}$; opx-sp.D = orthopyroxene-bearing spinel dunite; sp.H = spinel harzburgite; sp.L = spinel lherzolite. The "Remarks" column lists intrusive relations. 
Table 2. Average chemical composition of olivine in the MARK peridotites from Hole 920D.

\begin{tabular}{|c|c|c|c|c|c|c|c|c|c|c|c|c|}
\hline Core, section: & $2 \mathrm{R}-1$ & 11R-1 & $11 \mathrm{R}-1$ & $14 \mathrm{R}-3$ & $22 \mathrm{R}-2$ & $22 \mathrm{R}-2$ & $22 \mathrm{R}-3$ & $22 \mathrm{R}-4$ & $22 \mathrm{R}-5$ & $22 \mathrm{R}-5$ & $22 \mathrm{R}-6$ & $22 \mathrm{R}-7$ \\
\hline Piece no.: & 4 & 2 & 2 & 1 & $1 \mathrm{~A}$ & ID & $1 \mathrm{~A}$ & 4 & 3 & $6 \mathrm{C}$ & 12 & 4 \\
\hline Rock type: & opx-sp.D & opx-sp.D & sp.H & sp.H & sp.L & sp.L & sp.H & sp.H & sp.H & sp.H & sp.H & sp.H \\
\hline Analysis no.: & ol-1 & ol-2 & ol-3 & ol-4 & ol-5 & ol- 6 & ol-7 & ol-8 & ol-9 & ol- 10 & ol-11 & ol-12 \\
\hline No. averaged: & 11 & 3 & 10 & 19 & 15 & 16 & 13 & 12 & 18 & 29 & 15 & 22 \\
\hline $\begin{array}{c}\mathrm{SiO}_{2} \\
\mathrm{NiO} \\
\mathrm{FeO}^{*} \\
\mathrm{MnO} \\
\mathrm{MgO} \\
\mathrm{CaO} \\
\text { Total }\end{array}$ & $\begin{array}{r}40.90 \\
0.41 \\
9.02 \\
0.11 \\
50.20 \\
0.03 \\
100.67\end{array}$ & $\begin{array}{r}40.92 \\
0.26 \\
9.70 \\
0.10 \\
50.05 \\
0.00 \\
101.02\end{array}$ & $\begin{array}{r}41.21 \\
0.31 \\
9.20 \\
0.11 \\
50.26 \\
0.01 \\
101.09\end{array}$ & $\begin{array}{r}41.04 \\
0.37 \\
8.92 \\
0.11 \\
50.36 \\
0.04 \\
100.83\end{array}$ & $\begin{array}{r}41.02 \\
0.37 \\
8.85 \\
0.09 \\
50.50 \\
0.03 \\
100.86\end{array}$ & $\begin{array}{r}40.48 \\
0.35 \\
8.79 \\
0.13 \\
49.64 \\
0.03 \\
99.41\end{array}$ & $\begin{array}{r}41.24 \\
0.34 \\
8.77 \\
0.12 \\
50.67 \\
0.03 \\
101.17\end{array}$ & $\begin{array}{r}41.01 \\
0.34 \\
8.85 \\
0.06 \\
50.57 \\
0.01 \\
100.84\end{array}$ & $\begin{array}{r}41.34 \\
0.34 \\
8.96 \\
0.12 \\
50.43 \\
0.07 \\
101.26\end{array}$ & $\begin{array}{r}40.91 \\
0.38 \\
9.51 \\
0.13 \\
49.73 \\
0.03 \\
100.70\end{array}$ & $\begin{array}{r}41.18 \\
0.37 \\
8.91 \\
0.11 \\
50.64 \\
0.03 \\
101.24\end{array}$ & $\begin{array}{r}41.20 \\
0.33 \\
9.23 \\
0.11 \\
50.27 \\
0.02 \\
101.16\end{array}$ \\
\hline $\begin{array}{c}(\mathrm{O}=4) \\
\mathrm{Si} \\
\mathrm{Ni} \\
\mathrm{Fe} \\
\mathrm{Mn} \\
\mathrm{Mg} \\
\mathrm{Ca} \\
\text { Total }\end{array}$ & $\begin{array}{l}0.994 \\
0.008 \\
0.183 \\
0.002 \\
1.818 \\
0.001 \\
3.006\end{array}$ & $\begin{array}{l}0.993 \\
0.005 \\
0.197 \\
0.002 \\
1.810 \\
0.000 \\
3.007\end{array}$ & $\begin{array}{l}0.997 \\
0.006 \\
0.186 \\
0.002 \\
1.812 \\
0.000 \\
3.003\end{array}$ & $\begin{array}{l}0.995 \\
0.007 \\
0.181 \\
0.002 \\
1.819 \\
0.001 \\
3.005\end{array}$ & & & $\begin{array}{l}0.995 \\
0.007 \\
0.177 \\
0.002 \\
1.823 \\
0.001 \\
3.005\end{array}$ & & $\begin{array}{l}0.997 \\
0.007 \\
0.181 \\
0.003 \\
1.813 \\
0.002 \\
3.003\end{array}$ & $\begin{array}{l}0.996 \\
0.007 \\
0.194 \\
0.003 \\
1.804 \\
0.001 \\
3.004\end{array}$ & $\begin{array}{l}0.994 \\
0.007 \\
0.180 \\
0.002 \\
1.822 \\
0.001 \\
3.006\end{array}$ & $\begin{array}{l}0.996 \\
0.007 \\
0.187 \\
0.002 \\
1.812 \\
0.001 \\
3.004\end{array}$ \\
\hline Mg\# & 90.8 & 90.2 & 90.7 & 91.0 & 91.0 & 91.0 & 91.1 & 91.1 & 90.9 & 90.3 & 91.0 & 90.7 \\
\hline
\end{tabular}

Notes: $\mathrm{FeO}^{*}=$ total iron as $\mathrm{FeO} . \mathrm{Mg} \#=100 \mathrm{Mg} /(\mathrm{Mg}+\mathrm{Fe})$. Abbreviations as in Table 1.

Table 3. Average chemical composition of spinel in the MARK peridotites from Hole 920D.

\begin{tabular}{|c|c|c|c|c|c|c|c|c|c|c|c|c|}
\hline Core, section: & $2 \mathrm{R}-1$ & $11 \mathrm{R}-1$ & $11 \mathrm{R}-1$ & $14 \mathrm{R}-3$ & $22 \mathrm{R}-2$ & $22 \mathrm{R}-2$ & $22 \mathrm{R}-3$ & $22 \mathrm{R}-4$ & $22 \mathrm{R}-5$ & $22 R-5$ & $22 \mathrm{R}-6$ & $22 \mathrm{R}-7$ \\
\hline Piece no.: & 4 & 2 & 2 & 1 & $1 \mathrm{~A}$ & ID & $1 \mathrm{~A}$ & 4 & 3 & $6 \mathrm{C}$ & 12 & 4 \\
\hline Rock type: & opx-sp.D & opx-sp.D & sp.H & sp.H & sp.L & sp.L & sp.H & sp.H & sp.H & sp.H & sp.H & sp.H \\
\hline No. averaged: & 15 & 30 & 15 & 26 & 20 & 20 & 19 & 19 & 37 & 18 & 8 & 8 \\
\hline $\begin{array}{c}\mathrm{SiO}_{2} \\
\mathrm{TiO}_{2} \\
\mathrm{Al}_{2} \mathrm{O}_{3} \\
\mathrm{Cr}_{2} \mathrm{O}_{3} \\
\mathrm{NiO} \\
\mathrm{FeO}^{*} \\
\mathrm{MnO} \\
\mathrm{MgO} \\
\text { Total }\end{array}$ & $\begin{array}{r}0.00 \\
0.10 \\
40.27 \\
28.04 \\
0.25 \\
14.95 \\
0.20 \\
16.12 \\
99.93\end{array}$ & $\begin{array}{r}0.03 \\
0.18 \\
34.91 \\
32.62 \\
0.13 \\
17.05 \\
0.21 \\
14.95 \\
100.08\end{array}$ & $\begin{array}{r}0.02 \\
0.11 \\
37.19 \\
31.37 \\
0.14 \\
15.25 \\
0.19 \\
15.83 \\
100.08\end{array}$ & $\begin{array}{r}0.00 \\
0.11 \\
42.17 \\
26.52 \\
0.26 \\
13.47 \\
0.17 \\
17.55 \\
100.24\end{array}$ & $\begin{array}{r}0.01 \\
0.08 \\
42.08 \\
26.26 \\
0.24 \\
13.50 \\
0.14 \\
17.50 \\
99.83\end{array}$ & $\begin{array}{r}0.01 \\
0.11 \\
41.91 \\
26.18 \\
0.22 \\
13.52 \\
0.18 \\
17.32 \\
99.44\end{array}$ & $\begin{array}{r}0.04 \\
0.08 \\
42.63 \\
26.52 \\
0.21 \\
13.31 \\
0.17 \\
17.63 \\
100.59\end{array}$ & $\begin{array}{r}0.03 \\
0.06 \\
42.60 \\
25.41 \\
0.20 \\
14.08 \\
0.12 \\
17.34 \\
99.85\end{array}$ & $\begin{array}{r}0.04 \\
0.08 \\
42.56 \\
25.96 \\
0.24 \\
14.11 \\
0.19 \\
17.29 \\
100.46\end{array}$ & $\begin{array}{r}0.01 \\
0.22 \\
30.22 \\
37.05 \\
0.20 \\
17.73 \\
0.25 \\
14.46 \\
100.13\end{array}$ & $\begin{array}{r}0.03 \\
0.32 \\
28.02 \\
39.72 \\
0.19 \\
17.86 \\
0.25 \\
14.18 \\
100.56\end{array}$ & $\begin{array}{r}0.02 \\
0.04 \\
43.10 \\
25.44 \\
0.19 \\
13.92 \\
0.17 \\
17.45 \\
100.33\end{array}$ \\
\hline \multicolumn{13}{|l|}{$(\mathrm{O}=4)$} \\
\hline $\begin{array}{l}\mathrm{Mg \#} \\
\mathrm{Cr} \# \\
\text { Ferric\# } \\
\mathrm{Y}(\mathrm{Al}) \\
\mathrm{Y}(\mathrm{Cr}) \\
\mathrm{Y}\left(\mathrm{Fe}^{3+}\right)\end{array}$ & $\begin{array}{l}67.9 \\
32.0 \\
9.7 \\
0.67 \\
0.31 \\
0.02\end{array}$ & $\begin{array}{c}64.5 \\
38.5 \\
14.0 \\
0.60 \\
0.37 \\
0.03\end{array}$ & $\begin{array}{c}67.6 \\
36.1 \\
10.9 \\
0.63 \\
0.35 \\
0.02\end{array}$ & $\begin{array}{l}72.8 \\
29.6 \\
13.1 \\
0.69 \\
0.29 \\
0.02\end{array}$ & $\begin{array}{l}72.8 \\
29.5 \\
13.7 \\
0.69 \\
0.29 \\
0.02\end{array}$ & $\begin{array}{l}72.4 \\
29.5 \\
12.8 \\
0.69 \\
0.29 \\
0.02\end{array}$ & $\begin{array}{c}72.7 \\
29.4 \\
11.4 \\
0.69 \\
0.29 \\
0.02\end{array}$ & $\begin{array}{c}72.0 \\
28.5 \\
14.6 \\
0.70 \\
0.28 \\
0.02\end{array}$ & $\begin{array}{c}71.6 \\
29.0 \\
13.5 \\
0.69 \\
0.28 \\
0.02\end{array}$ & $\begin{array}{c}63.8 \\
45.1 \\
17.6 \\
0.53 \\
0.43 \\
0.04\end{array}$ & $\begin{array}{c}62.9 \\
48.7 \\
16.5 \\
0.49 \\
0.47 \\
0.04\end{array}$ & $\begin{array}{c}72.1 \\
28.3 \\
13.4 \\
0.70 \\
0.28 \\
0.02\end{array}$ \\
\hline
\end{tabular}

Notes: $\mathrm{FeO}^{*}=$ total iron as $\mathrm{FeO} . \mathrm{Fe}^{3+}$ calculation on the basis of $\mathrm{AB}_{2} \mathrm{O}_{4}$ stoichiometry. $\mathrm{Mg} \#=100 \mathrm{Mg} /(\mathrm{Mg}+\mathrm{Fe}), \mathrm{Cr} \#=100 \mathrm{Cr} /(\mathrm{Cr}+\mathrm{Al}), \mathrm{Ferric} \#=100 \mathrm{Fe}{ }^{3+} /\left(\mathrm{Fe}{ }^{3+}+\mathrm{Fe}{ }^{2+}\right), \mathrm{Abbrevia}^{-}$ tions as in Table 1.

$=0.37 \mathrm{wt} \%$. More than $95 \%$ of the analyses ( 180 olivine grains) from the 10 samples examined plot in a small compositional range. No systematic variations in Fo contents and $\mathrm{NiO}$ wt\% with modal abundances of pyroxenes were found in the range of rock types from lherzolite to pyroxene-poor harzburgite.

There are some deviations from the cluster of primary olivine compositions that are rich in $\mathrm{FeO}^{*}(*=$ total iron as $\mathrm{FeO}$ ) (Fig. 1). The olivines enriched in $\mathrm{FeO} *(\mathrm{Fo}=87.5-89.5)$ are limited to small portions of Samples 153-920D-22R-5 (Piece 6C, 120-128 cm) and 22R-7 (Piece 4, 56-61 cm), both of which are in contact with pieces crosscut by oxide-rich gabbro and pyroxenite intrusive rocks. Other deviations are also detectable as $\mathrm{NiO}$ depletion with $\mathrm{FeO}^{*}$ enrichment within the orthopyroxene-bearing spinel dunite (Sample 153920D-11R-1, Piece 2, 15-19 cm), in which the olivine cores are poor- er both in Fo and $\mathrm{NiO}$ contents than those in the spinel harzburgites and lherzolites (Table 2).

\section{Spinel}

Spinels in the MARK peridotites from Hole 920D are yellowish brown in harzburgites and lherzolites to brown in orthopyroxenebearing spinel dunites. The $\mathrm{Mg} \#$ value $(\mathrm{Mg} \#=100 \mathrm{Mg} /[\mathrm{Mg}+\mathrm{Fe}])$ of spinel cores in the spinel harzburgites and lherzolites varies within the compositional range for upper mantle peridotites from 56.6 to 74.3 . The $\mathrm{Cr} \#$ value $(\mathrm{Cr} \#=100 \mathrm{Cr} /[\mathrm{Cr}+\mathrm{Al}])$ of spinel cores ranges widely from 27.2 to 52.2 . As shown in Figure $2 \mathrm{~A}$, the $\mathrm{Mg \#}$ and $\mathrm{Cr} \#$ values of spinel cores plot in the compositional ranges defined by spinels from the Mid-Atlantic Ridge areas (Prinz et al., 1976; 
Table 4. Average chemical composition of orthopyroxene in the MARK peridotites from Hole 920D.

\begin{tabular}{|c|c|c|c|c|c|c|c|c|c|c|c|c|}
\hline Core, section: & $2 \mathrm{R}-1$ & $11 \mathrm{R}-1$ & $11 \mathrm{R}-1$ & $14 \mathrm{R}-3$ & 22R-2 & $22 \mathrm{R}-2$ & $22 \mathrm{R}-3$ & $22 R-4$ & $22 \mathrm{R}-5$ & 22R-5 & $22 \mathrm{R}-6$ & $22 \mathrm{R}-7$ \\
\hline Piece no.: & 4 & 2 & 2 & 1 & $1 \mathrm{~A}$ & ID & $1 \mathrm{~A}$ & 4 & 3 & $6 \mathrm{C}$ & 12 & 4 \\
\hline Rock type: & opx-sp.D & opx-sp.D & sp.H & sp.H & sp.L & sp.L & sp.H & sp.H & sp.H & sp.H & $\mathrm{sp} . \mathrm{H}$ & sp.H \\
\hline Analysis no.: & opx-1 & opx-2 & opx-3 & opx -4 & opx-5 & орx-6 & opx-7 & opx -8 & opx-9 & opx-10 & opx-11 & opx-12 \\
\hline No. averaged: & 4 & 4 & 6 & 13 & 3 & 8 & 8 & 12 & 7 & 12 & 6 & 9 \\
\hline $\begin{array}{c}\mathrm{SiO}_{2} \\
\mathrm{TiO}_{2} \\
\mathrm{Al}_{2} \mathrm{O}_{3} \\
\mathrm{Cr}_{2} \mathrm{O}_{3} \\
\mathrm{NiO} \\
\mathrm{FeO} \\
\mathrm{MnO} \\
\mathrm{MgO} \\
\mathrm{CaO} \\
\mathrm{Na}_{2} \mathrm{O} \\
\text { Total }\end{array}$ & $\begin{array}{r}55.28 \\
0.03 \\
3.37 \\
0.71 \\
0.11 \\
5.75 \\
0.12 \\
33.20 \\
1.12 \\
0.03 \\
99.72\end{array}$ & $\begin{array}{r}55.38 \\
0.01 \\
3.16 \\
0.65 \\
0.01 \\
6.09 \\
0.13 \\
32.61 \\
1.61 \\
0.00 \\
99.64\end{array}$ & $\begin{array}{r}55.52 \\
0.01 \\
3.35 \\
0.72 \\
0.02 \\
5.95 \\
0.11 \\
32.78 \\
1.67 \\
0.01 \\
100.15\end{array}$ & $\begin{array}{r}55.30 \\
0.04 \\
3.74 \\
0.74 \\
0.08 \\
5.78 \\
0.11 \\
33.03 \\
1.42 \\
0.01 \\
100.25\end{array}$ & $\begin{array}{r}55.01 \\
0.04 \\
3.84 \\
0.76 \\
0.07 \\
5.74 \\
0.10 \\
32.81 \\
1.84 \\
0.00 \\
100.21\end{array}$ & $\begin{array}{r}54.70 \\
0.05 \\
3.80 \\
0.73 \\
0.05 \\
5.61 \\
0.13 \\
32.40 \\
1.56 \\
0.01 \\
99.04\end{array}$ & $\begin{array}{r}55.63 \\
0.00 \\
3.75 \\
0.68 \\
0.03 \\
5.58 \\
0.11 \\
32.86 \\
1.79 \\
0.00 \\
100.43\end{array}$ & $\begin{array}{r}55.03 \\
0.01 \\
3.97 \\
0.75 \\
0.04 \\
5.76 \\
0.08 \\
32.99 \\
1.29 \\
0.00 \\
99.91\end{array}$ & $\begin{array}{r}55.64 \\
0.00 \\
4.06 \\
0.80 \\
0.04 \\
5.96 \\
0.13 \\
32.81 \\
1.48 \\
0.00 \\
100.92\end{array}$ & $\begin{array}{r}55.05 \\
0.05 \\
3.51 \\
0.81 \\
0.06 \\
6.27 \\
0.14 \\
32.89 \\
1.12 \\
0.00 \\
99.89\end{array}$ & $\begin{array}{r}55.96 \\
0.07 \\
2.90 \\
0.75 \\
0.08 \\
5.84 \\
0.13 \\
33.68 \\
1.20 \\
0.01 \\
100.63\end{array}$ & $\begin{array}{r}55.62 \\
0.01 \\
3.83 \\
0.73 \\
0.06 \\
5.72 \\
0.11 \\
33.10 \\
1.59 \\
0.00 \\
100.77\end{array}$ \\
\hline \multicolumn{13}{|l|}{$(\mathrm{O}=6)$} \\
\hline $\begin{array}{c}\mathrm{Si} \\
\mathrm{Ti} \\
\mathrm{Al} \\
\mathrm{Cr} \\
\mathrm{Ni} \\
\mathrm{Fe} \\
\mathrm{Mn} \\
\mathrm{Mg} \\
\mathrm{Ca} \\
\mathrm{Na} \\
\text { Total }\end{array}$ & $\begin{array}{l}1.916 \\
0.001 \\
0.138 \\
0.019 \\
0.003 \\
0.167 \\
0.003 \\
1.715 \\
0.042 \\
0.002 \\
4.006\end{array}$ & $\begin{array}{l}1.924 \\
0.000 \\
0.130 \\
0.018 \\
0.000 \\
0.177 \\
0.004 \\
1.689 \\
0.060 \\
0.000 \\
4.002\end{array}$ & $\begin{array}{l}1.919 \\
0.000 \\
0.137 \\
0.020 \\
0.001 \\
0.172 \\
0.003 \\
1.689 \\
0.062 \\
0.001 \\
4.003\end{array}$ & $\begin{array}{l}1.908 \\
0.001 \\
0.153 \\
0.020 \\
0.002 \\
0.167 \\
0.003 \\
1.698 \\
0.053 \\
0.001 \\
4.005\end{array}$ & $\begin{array}{l}1.901 \\
0.001 \\
0.157 \\
0.021 \\
0.002 \\
0.166 \\
0.003 \\
1.690 \\
0.068 \\
0.000 \\
4.009\end{array}$ & $\begin{array}{l}1.905 \\
0.001 \\
0.157 \\
0.020 \\
0.001 \\
0.164 \\
0.004 \\
1.693 \\
0.059 \\
0.000 \\
4.005\end{array}$ & $\begin{array}{l}1.914 \\
0.000 \\
0.152 \\
0.018 \\
0.001 \\
0.161 \\
0.003 \\
1.685 \\
0.066 \\
0.000 \\
4.000\end{array}$ & $\begin{array}{l}1.903 \\
0.000 \\
0.162 \\
0.021 \\
0.001 \\
0.167 \\
0.002 \\
1.701 \\
0.048 \\
0.000 \\
4.005\end{array}$ & $\begin{array}{l}1.907 \\
0.000 \\
0.164 \\
0.022 \\
0.001 \\
0.171 \\
0.004 \\
1.676 \\
0.054 \\
0.000 \\
4.000\end{array}$ & $\begin{array}{l}1.909 \\
0.001 \\
0.144 \\
0.022 \\
0.002 \\
0.182 \\
0.004 \\
1.700 \\
0.041 \\
0.000 \\
4.006\end{array}$ & $\begin{array}{l}1.923 \\
0.002 \\
0.118 \\
0.020 \\
0.002 \\
0.168 \\
0.004 \\
1.725 \\
0.044 \\
0.001 \\
4.007\end{array}$ & $\begin{array}{l}1.908 \\
0.000 \\
0.155 \\
0.020 \\
0.002 \\
0.164 \\
0.003 \\
1.693 \\
0.058 \\
0.000 \\
4.004\end{array}$ \\
\hline $\begin{array}{l}\mathrm{Mg \#} \\
\mathrm{Cr} \#\end{array}$ & $\begin{array}{l}91.1 \\
12.3\end{array}$ & $\begin{array}{l}90.5 \\
12.1\end{array}$ & $\begin{array}{l}90.8 \\
12.6\end{array}$ & $\begin{array}{l}91.1 \\
11.6\end{array}$ & $\begin{array}{l}91.1 \\
11.7\end{array}$ & $\begin{array}{l}91.1 \\
11.4\end{array}$ & $\begin{array}{l}91.3 \\
10.8\end{array}$ & $\begin{array}{l}91.1 \\
11.2\end{array}$ & $\begin{array}{l}90.8 \\
11.7\end{array}$ & $\begin{array}{l}90.3 \\
13.5\end{array}$ & $\begin{array}{l}91.1 \\
15.1\end{array}$ & $\begin{array}{l}91.2 \\
11.2\end{array}$ \\
\hline
\end{tabular}

Notes: $\mathrm{FeO}^{*}=$ total iron as $\left.\mathrm{FeO} \cdot \mathrm{Mg} \#=100 \mathrm{Mg} / \mathrm{Mg}+\mathrm{Fe}\right), \mathrm{Cr} \#=100 \mathrm{Cr} /(\mathrm{Cr}+\mathrm{Al})$. Abbreviations as in Table 1 .

Table 5. Average chemical composition of clinopyroxene in the MARK peridotites from Hole 920D.

\begin{tabular}{|c|c|c|c|c|c|c|c|c|c|}
\hline Core, section: & $14 \mathrm{R}-3$ & $22 \mathrm{R}-2$ & $22 \mathrm{R}-2$ & $22 \mathrm{R}-3$ & $22 \mathrm{R}-4$ & $22 \mathrm{R}-5$ & $22 \mathrm{R}-5$ & $22 R-6$ & $22 \mathrm{R}-7$ \\
\hline Piece no.: & 1 & $1 \mathrm{~A}$ & ID & $1 \mathrm{~A}$ & 4 & 3 & $6 \mathrm{C}$ & 12 & 4 \\
\hline Rock type: & sp.H & sp.L & sp.L & sp.H & sp.H & sp.H & sp.H & sp.H & sp.H \\
\hline Analysis no.: & $\operatorname{cpx}-2$ & $\operatorname{cpx}-3$ & $\operatorname{cpx}-4$ & cpx-5 & cpx-6 & cpx-7 & cpx-8 & cpx-9 & cpx-10 \\
\hline No. averaged: & 12 & 17 & 12 & 9 & 19 & 11 & 23 & 15 & 11 \\
\hline $\begin{array}{l}\mathrm{SiO}_{2} \\
\mathrm{TiO}_{2} \\
\mathrm{Al}_{2} \mathrm{O}_{3} \\
\mathrm{Cr}_{2} \mathrm{O}_{3} \\
\mathrm{NiO} \\
\mathrm{FeO} \\
\mathrm{MnO} \\
\mathrm{MgO} \\
\mathrm{CaO} \\
\stackrel{\mathrm{Na}}{2} \mathrm{O} \\
\text { Total }\end{array}$ & $\begin{array}{r}51.33 \\
0.10 \\
5.01 \\
1.28 \\
0.05 \\
2.49 \\
0.08 \\
16.38 \\
23.20 \\
0.08 \\
99.99\end{array}$ & $\begin{array}{r}51.53 \\
0.09 \\
4.73 \\
1.18 \\
0.02 \\
2.35 \\
0.03 \\
16.50 \\
23.29 \\
0.07 \\
99.80\end{array}$ & $\begin{array}{r}50.82 \\
0.12 \\
4.72 \\
1.15 \\
0.03 \\
2.40 \\
0.09 \\
16.34 \\
23.06 \\
0.08 \\
98.81\end{array}$ & $\begin{array}{r}51.89 \\
0.06 \\
4.69 \\
1.11 \\
0.01 \\
2.46 \\
0.08 \\
17.14 \\
22.84 \\
0.07 \\
100.36\end{array}$ & $\begin{array}{r}51.27 \\
0.09 \\
4.84 \\
1.20 \\
0.01 \\
2.29 \\
0.03 \\
16.32 \\
23.55 \\
0.05 \\
99.66\end{array}$ & $\begin{array}{r}51.66 \\
0.06 \\
5.17 \\
1.28 \\
0.02 \\
2.64 \\
0.10 \\
16.65 \\
22.70 \\
0.06 \\
100.33\end{array}$ & $\begin{array}{r}51.63 \\
0.13 \\
4.37 \\
1.26 \\
0.07 \\
2.53 \\
0.09 \\
16.49 \\
23.25 \\
0.04 \\
99.86\end{array}$ & $\begin{array}{r}51.78 \\
0.21 \\
4.45 \\
1.38 \\
0.05 \\
2.49 \\
0.08 \\
16.50 \\
23.09 \\
0.16 \\
100.19\end{array}$ & $\begin{array}{r}51.81 \\
0.05 \\
4.79 \\
1.13 \\
0.03 \\
2.56 \\
0.08 \\
16.77 \\
23.08 \\
0.05 \\
100.36\end{array}$ \\
\hline \multicolumn{10}{|l|}{$(\mathrm{O}=6)$} \\
\hline $\begin{array}{c}\mathrm{Si} \\
\mathrm{Ti} \\
\mathrm{Al} \\
\mathrm{Cr} \\
\mathrm{Ni} \\
\mathrm{Fe} \\
\mathrm{Mn} \\
\mathrm{Mg} \\
\mathrm{Ca} \\
\mathrm{Na} \\
\text { Total }\end{array}$ & $\begin{array}{l}1.870 \\
0.003 \\
0.216 \\
0.037 \\
0.001 \\
0.076 \\
0.002 \\
0.889 \\
0.906 \\
0.005 \\
4.004\end{array}$ & $\begin{array}{l}1.878 \\
0.002 \\
0.204 \\
0.034 \\
0.001 \\
0.072 \\
0.001 \\
0.897 \\
0.910 \\
0.005 \\
4.003\end{array}$ & $\begin{array}{l}1.873 \\
0.003 \\
0.206 \\
0.033 \\
0.001 \\
0.074 \\
0.003 \\
0.897 \\
0.911 \\
0.006 \\
4.007\end{array}$ & $\begin{array}{l}1.879 \\
0.002 \\
0.201 \\
0.032 \\
0.000 \\
0.074 \\
0.003 \\
0.925 \\
0.886 \\
0.005 \\
4.006\end{array}$ & $\begin{array}{l}1.873 \\
0.002 \\
0.209 \\
0.035 \\
0.000 \\
0.070 \\
0.001 \\
0.889 \\
0.922 \\
0.004 \\
4.005\end{array}$ & $\begin{array}{l}1.872 \\
0.002 \\
0.221 \\
0.037 \\
0.000 \\
0.080 \\
0.003 \\
0.899 \\
0.882 \\
0.004 \\
4.000\end{array}$ & $\begin{array}{l}1.884 \\
0.003 \\
0.188 \\
0.036 \\
0.002 \\
0.077 \\
0.003 \\
0.897 \\
0.909 \\
0.003 \\
4.002\end{array}$ & $\begin{array}{l}1.882 \\
0.006 \\
0.191 \\
0.040 \\
0.002 \\
0.076 \\
0.003 \\
0.894 \\
0.899 \\
0.011 \\
4.003\end{array}$ & $\begin{array}{l}1.878 \\
0.001 \\
0.205 \\
0.032 \\
0.001 \\
0.078 \\
0.002 \\
0.906 \\
0.897 \\
0.003 \\
4.003\end{array}$ \\
\hline $\begin{array}{l}\mathrm{Mg \#} \\
\mathrm{Cl}^{\mathrm{II}} \\
\mathrm{Al}^{\mathrm{V}} / \mathrm{Al}^{\mathrm{IV}}\end{array}$ & $\begin{array}{c}92.1 \\
14.6 \\
0.65\end{array}$ & $\begin{array}{c}92.6 \\
14.2 \\
0.67\end{array}$ & $\begin{array}{c}92.4 \\
13.9 \\
0.62\end{array}$ & $\begin{array}{c}92.6 \\
13.7 \\
0.66\end{array}$ & $\begin{array}{c}92.7 \\
14.1 \\
0.65\end{array}$ & $\begin{array}{c}91.8 \\
14.2 \\
0.73\end{array}$ & $\begin{array}{c}92.1 \\
16.3 \\
0.62\end{array}$ & $\begin{array}{l}92.2 \\
17.3 \\
0.62\end{array}$ & $\begin{array}{c}92.1 \\
13.6 \\
0.69\end{array}$ \\
\hline
\end{tabular}

Notes: $\mathrm{FeO}^{*}=$ total iron as $\mathrm{FeO} \cdot \mathrm{Mg} \#=100 \mathrm{Mg} /(\mathrm{Mg}+\mathrm{Fe}), \mathrm{Cr} \#=100 \mathrm{Cr} /(\mathrm{Cr}+\mathrm{Al})$. Abbreviations as in Table 1.

Clarke and Loubat, 1977; Sigurdsson, 1977; Symes et al., 1977; Arai and Fujii, 1979; Sinton, 1979; Fujii, 1990; Juteau et al., 1990; Komor et al., 1990; Bonatti et al., 1992; Cannat et al., 1992). A highly uniform composition of primary spinel cores $(\mathrm{Mg} \#=70-75$ and $\mathrm{Cr} \#=27-31$ ) is conspicuous in Hole $920 \mathrm{D}$ spinel harzburgites and lherzolites (Fig. 2B).
Spinels from three samples have higher $\mathrm{Cr} \#$ values: $\mathrm{Cr} \#=36$ (153-920D-11R-1, Piece 2, 11-15 cm), Cr\# = 45 (153-920D-22R-5, Piece 6C, 120-128 cm), and Cr\# = 49 (153-920D-22R-6, Piece 12, $111-117 \mathrm{~cm}$ ). These spinels also have higher $\mathrm{FeO}^{*}$ and $\mathrm{TiO}_{2}$ contents than those of the primary spinels with $\mathrm{Cr} \#=27-31$. In particular, spinels in Samples 153-920D-22R-5 (Piece 6C, 120-128 cm) and 


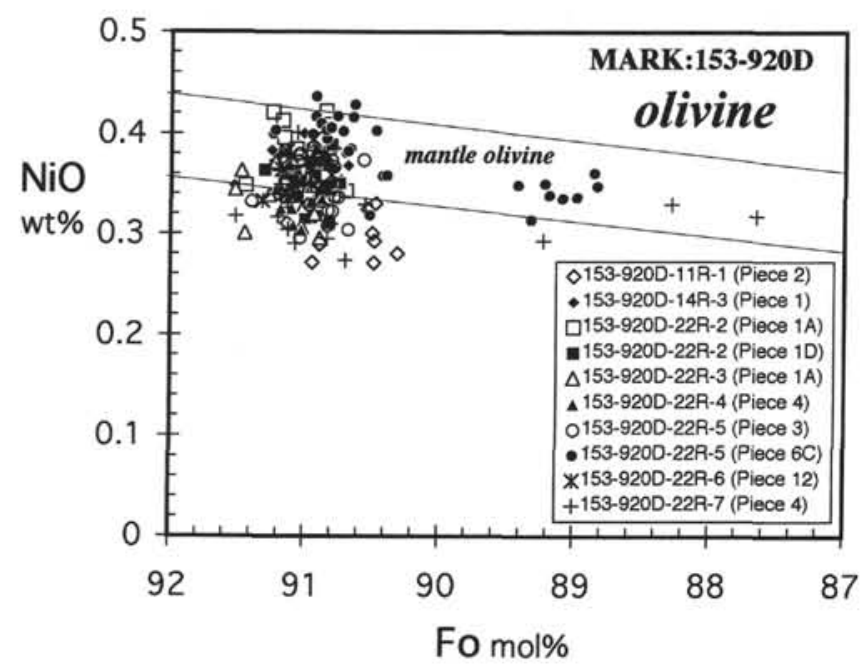

Figure 1. Plots of $\mathrm{NiO}$ (wt\%) vs. Fo content (mol\%) for olivine cores in the spinel harzburgites and lherzolites from Hole 920D. Note the highly concentration around $\mathrm{Fo}=91.0$ and $\mathrm{NiO}=0.37 \mathrm{wt} \%$. Modified olivines, enriched in FeO*, in Samples 153-920D-22R-5 (Piece 6C) and 153-920D-22R-7 (Piece 4 ), are plotted around Fo $=89-88$. Thin solid lines show the compositional range of mantle olivines defined by those in mantle-derived xenoliths (Takahashi et al., 1987).
153-920D-22R-6 (Piece 12, 111-117 cm) contain as much as 0.35 wt $\% \mathrm{TiO}_{2}$ and more than $0.03 \mathrm{Fe}^{3+} /\left(\mathrm{Fe}^{3+}+\mathrm{Al}+\mathrm{Cr}\right)$. As shown in the average composition of Table 3 , spinels in the orthopyroxene-bearing spinel dunites also have higher $\mathrm{FeO}^{*}(\mathrm{Mg} \#=53.8-72.0)$ and $\mathrm{TiO}_{2}$ contents $(0.05-0.22 \mathrm{wt} \%)$.

\section{Orthopyroxene}

The $\mathrm{Mg} \#$ of primary orthopyroxene cores in spinel harzburgites and therzolites ranges from 90.5 to 91.6 (Table 4). The orthopyroxenes have a higher range of $\mathrm{Al}_{2} \mathrm{O}_{3}$ contents from 3.17 to $4.77 \mathrm{wt} \%$, indicating the moderately depleted nature of the upper mantle peridotites. The eight samples of spinel harzburgites and lherzolites from Hole 920D contain compositionally uniform orthopyroxenes with an average composition of $\mathrm{Mg} \#=91.0$ and $\mathrm{Al}_{2} \mathrm{O}_{3}=3.8 \mathrm{wt} \%$ (Fig. 3). No systematic variations in $\mathrm{Mg} \#$ and $\mathrm{Al}_{2} \mathrm{O}_{3}$ with the modal variation of pyroxenes ( $15 \%$ to $32 \%$ as total pyroxenes) can be detected. Samples 153-920D-22R-5 (Piece 6C, 120-128 cm) and 153-920D-22R-6 (Piece 12, 111-117 cm) contain compositionally modified orthopyroxenes with depletion of $\mathrm{Al}_{2} \mathrm{O}_{3}$ with or without $\mathrm{FeO}$ * enrichment, as shown in Figure 3. Orthopyroxenes in the orthopyroxene-bearing spinel dunites also display similar depletions in $\mathrm{Mg} \#$ and $\mathrm{Al}_{2} \mathrm{O}_{3}$ (Table 4).

\section{Clinopyroxene}

The Mg\# of primary clinopyroxene cores in the spinel harzburgites and lherzolites from Hole 920D ranges from 91.5 to 93.4 (Table

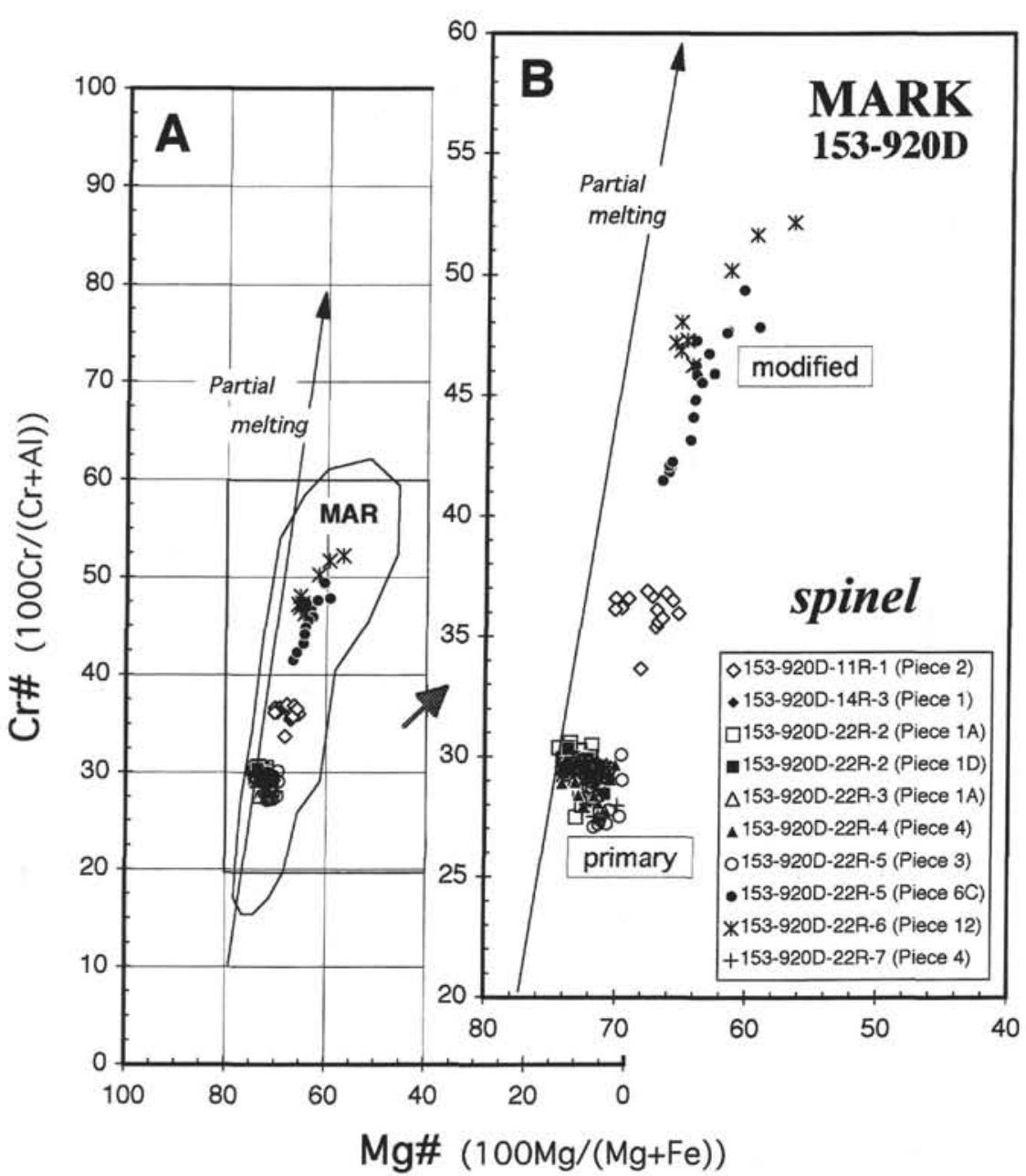

Figure 2. A. Plot of $\mathrm{Cr} \#(100 \mathrm{Cr} /[\mathrm{Cr}+\mathrm{Al}])$ vs. $\mathrm{Mg} \#$ $(100 \mathrm{Mg} /[\mathrm{Mg}+\mathrm{Fe}])$ for spinel cores in the spinel harzburgites and lherzolites from Hole 920D. All of the spinel analyses plot in the compositional range of spinels from the Mid-Atlantic Ridge area (see text for data sources). B. Expanded part of A. Note that the primary spinel cores form a highly concentrated cluster within the ranges of $\mathrm{Mg} \#=70-75$ and $\mathrm{Cr} \#=27-31$. Three samples (Samples 153-920D-11R-1, Piece 2; 22R-5, Piece 6C; and $22 \mathrm{R}-6$, Piece 12) contain modified spinels with higher $\mathrm{Cr} \#$ and $\mathrm{FeO}^{*}$. $\mathrm{MAR}=$ Mid-Atlantic Ridge. 
5). The $\mathrm{Al}_{2} \mathrm{O}_{3}$ contents vary within a slightly higher range (from 3.29 to $5.72 \mathrm{wt} \%$ ) than those of orthopyroxenes. The $\mathrm{TiO}_{2}$ contents are commonly less than $0.15 \mathrm{wt} \%$. The compositional ranges of the Hole 920D clinopyroxenes are comparable with those from Site 670 (Leg 109) in the MARK area (Fujii, 1990; Hébert et al., 1990; Juteau et al., 1990; Komor et al., 1990) and Site 395 (Deep Sea Drilling Project [DSDP] Leg 45) in the western flank of the Mid-Atlantic Ridge near the MARK area (Arai and Fujii, 1979; Sinton, 1979).

The Hole 920D clinopyroxenes are extremely poor in both $\mathrm{Ti}$ and $\mathrm{Na}(100 \mathrm{Ti}<0.7,100 \mathrm{Na}<1.2)$, suggesting a highly depleted peridotite signature, compared with other suboceanic peridotites (Fig. 4). Some compositionally modified clinopyroxenes observed in Hole $920 \mathrm{D}$ are enriched in $\mathrm{FeO}^{*}$ and $\mathrm{TiO}_{2}$. The maximum content of $\mathrm{TiO}_{2}$ attains $0.26 \mathrm{wt} \%$ in harzburgite (Sample 153-920D-22R-6, Piece 12,

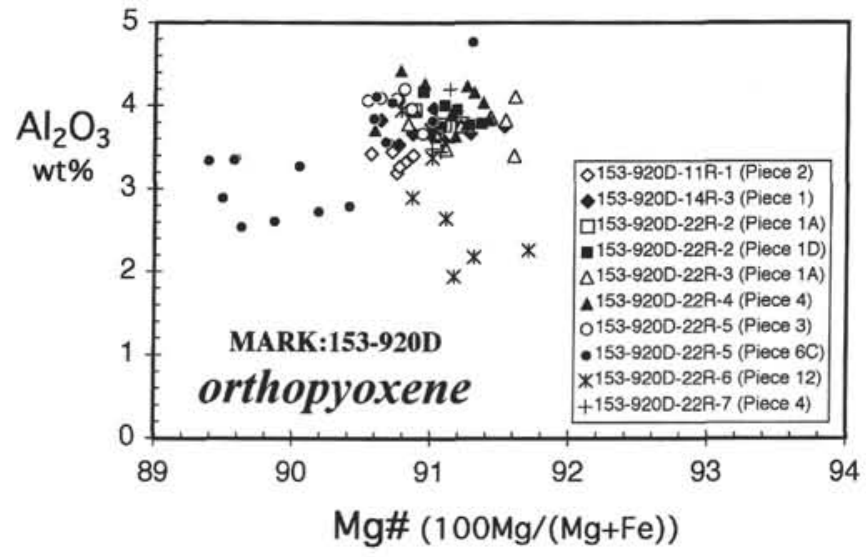

Figure 3. Plots of $\mathrm{Al}_{2} \mathrm{O}_{3}$ (wt\%) vs. $\mathrm{Mg} \#$ (100Mg/[Mg $\left.+\mathrm{Fe}\right]$ ) for orthopyroxene cores in spinel harzburgites and lherzolites from Hole 920D. Note the concentration around $\mathrm{Mg} \#=91.0 \mathrm{wt} \%$ and $\mathrm{Al}_{2} \mathrm{O}_{3}=3.8 \mathrm{wt} \%$. Samples 153 920D-22R-5 (Piece 6C) and 153-920D-22R-6 (Piece 12) contain $\mathrm{Al}_{2} \mathrm{O}_{3}$-poor orthopyroxenes with or without $\mathrm{FeO}^{*}$ enrichment.
$111-117 \mathrm{~cm})$. As shown in Figure 5, the $\mathrm{Al}^{\mathrm{VI}} / \mathrm{Al}^{\mathrm{IV}}$ ratios of the Hole 920D clinopyroxenes are notably low, ranging from 0.403 to 0.860 . They are lower than those of other suboceanic peridotites from the American-Antarctic Ridge, the Southwest Indian Ridge, and those of the pre-oceanic rifting-related peridotites from Saint Paul's Rocks, Galicia Margin, Zabargad Island in Red Sea, the ocean floor off southwestern Australia, and Antarctic Seamount B, suggesting shallow level equilibration.

\section{MODIFICATION OF PERIDOTITES BY MAGMA CHANNELING}

\section{Magma Channels in Harzburgite}

Several traces of magmas channeled through the upper mantle are observed as dikes and magmatic veins in the peridotite sections from Holes 920B and 920D (Cannat, Karson, Miller, et al., 1995). Two samples of spinel harzburgite (Piece 4 of Section 153-920D-10R-2, $35-41 \mathrm{~cm}$, and Piece 7 of Section 153-920D-13R-2, 136-143 cm) crosscut by websterite veins were examined here.

In Piece 4 of Section 153-920D-10R-2, 0.8-cm-thick replacement bands of modified spinel harzburgites are molded within the harzburgite wall in contact with 0.3 -cm-thick, oxide-rich, websterite vein. The wall harzburgite is severely altered into a low-temperature secondary assemblage of serpentine + magnetite. Small amounts of primary olivine, spinel, and orthopyroxene are preserved in the serpentinized harzburgite. In the replacement band, more than $75 \%$ of the primary grains of olivine, spinel, orthopyroxene, and clinopyroxene are well preserved, showing a porphyroclastic texture with large orthopyroxene and olivine porphyroclasts in a neoblastic matrix. The websterite vein, which is composed of clinopyroxene, orthopyroxene, olivine, ilmenite, and $\mathrm{Ni}-\mathrm{Fe}$ oxide, sharply contacts the modified spinel harzburgite along an irregular border surface.

In Piece 7 of Section 153-920D-13R-2, 2.0-cm-thick replacement bands of modified spinel harzburgite are molded within the harzburgite wall in contact with 4.0 -cm-thick websterite vein. Similar features
Figure 4. Plots of $100 \mathrm{Ti}$ vs. $100 \mathrm{Na}$ (atomic proportions for $\mathrm{O}=6$ ) for clinopyroxenes in suboceanic peridotites. The clinopyroxenes in the spinel harzburgites and lherzolites from Hole 920D are plotted in the lower left corner. The solid line shows various degrees of partial melting expected for the suboceanic peridotites, and the dashed line shows a trend of compositional modification through melt impregnation (Girardeau and Francheteau, 1993). Data sources for clinopyroxenes from Mid-Atlantic Ridge (Prinz et al., 1976; Clark and Loubat, 1977; Symes et al., 1977; Arai and Fujii, 1979; Sinton, 1979; Fujii, 1990; Hébert et al., 1990; Juteau et al., 1990; Komor et al., 1990; Bonatti et al., 1992; Cannat et al., 1992); American-Antarctic Ridge (AAR) and Southwest Indian Ridge (SWIR; Dick, 1989); and those related to the pre-oceanic rifting during the early stage of continental breakup (preoceanic rifts) from Saint Paul's Rocks (Melson et al., 1972), Galicia Margin (Evans and Girardeau, 1988; Kornprobst and Tabit, 1988), Zabargad (Bonatti et al., 1986; Piccardo et al., 1988), southwest Australia (Nicholls et al., 1981), and Antarctic Seamount B (Niida and Yuasa. 1995).

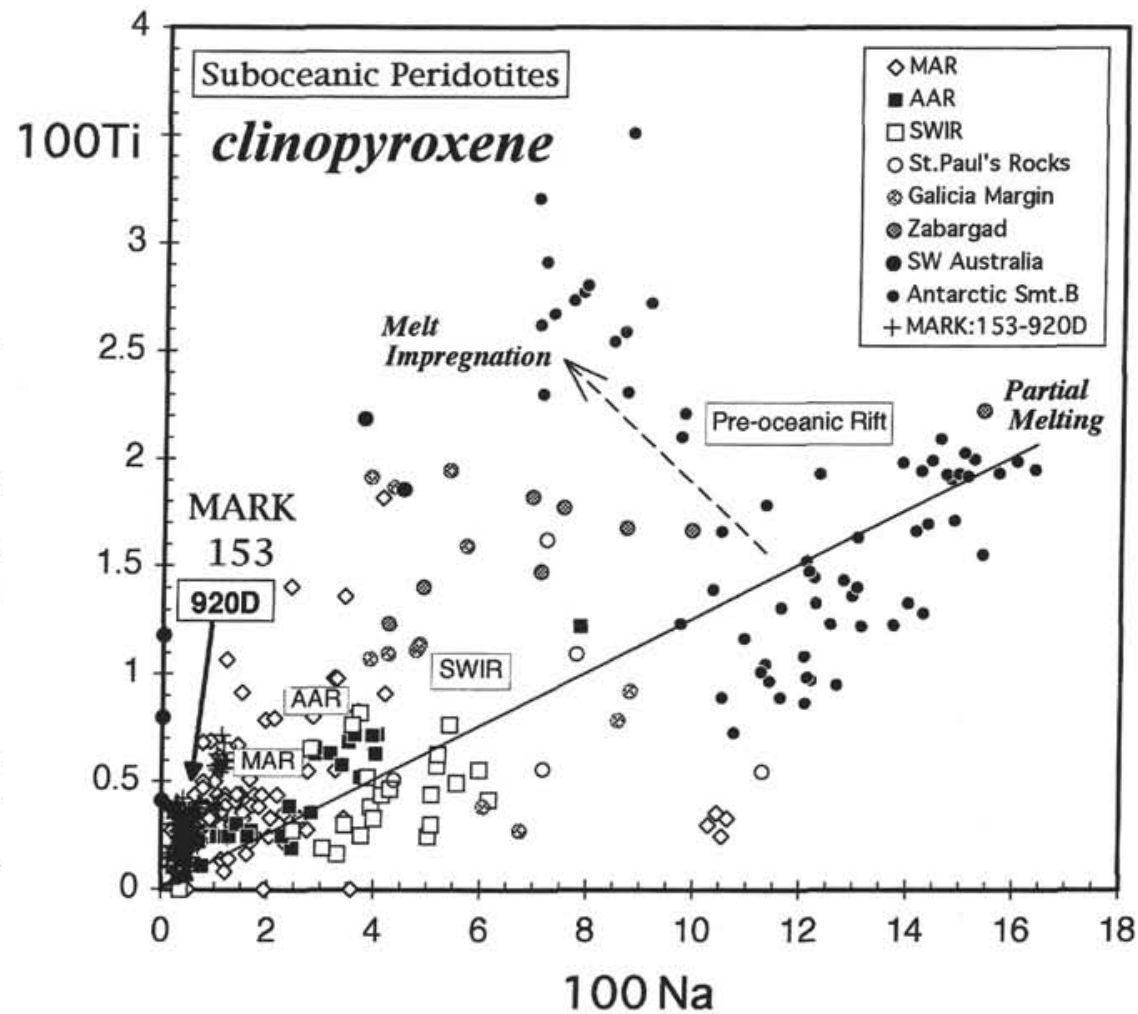




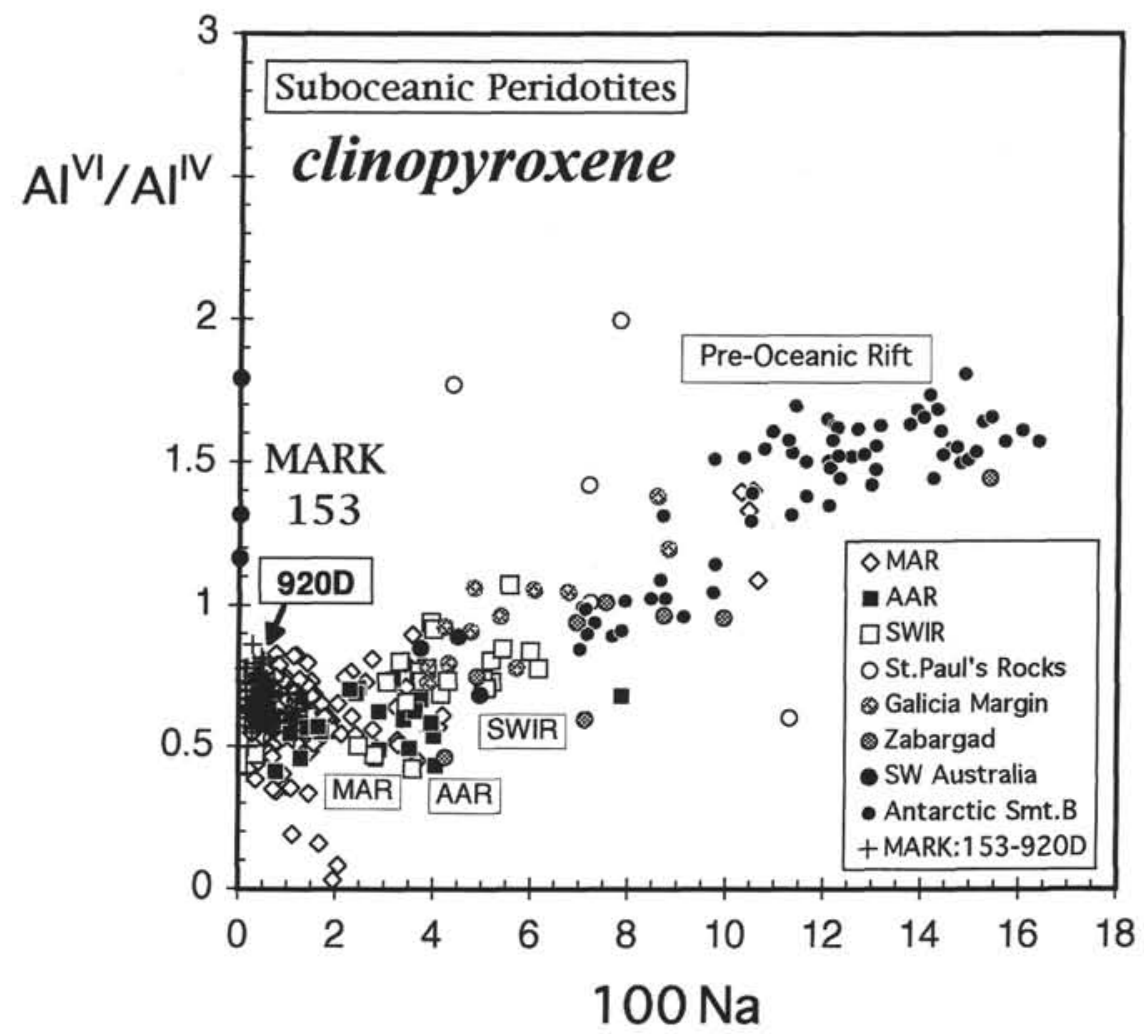

Figure 5. Plots of $\mathrm{Al}^{\mathrm{VI} / \mathrm{Al}^{\mathrm{IV}}}$ (atomic ratios) vs. $100 \mathrm{Na}$ (atomic proportions for $\mathrm{O}=6$ ) for clinopyroxenes in suboceanic peridotites. The clinopyroxenes in the spinel harzburgites and lherzolites from Hole 920D are plotted in the left bottom corner. Data sources for clinopyroxenes are the same as in Figure 4. of preservation and alteration in primary minerals are observed in the harzburgite. The websterite vein is composed mostly of orthopyroxene and clinopyroxene with small amounts of ilmenite and plagioclase.

\section{Variations in Chemical Composition Across Channels}

Variations in chemical composition of primary minerals were examined across channels in Piece 4 (35-41 cm) of Section 153-920D10R-2 and Piece 7 (136-143 cm) of Section 153-920D-13R-2. Microprobe data for cores of individual minerals were plotted vs. the distance $(\mathrm{mm})$ across channels in Figures 6 through 9. Minerals in the wall harzburgite have the "uniform" compositions of primary minerals described earlier (Figs. 1-5), whereas they show steep compositional gradients in the replacement bands.

In Piece 4 of Section 153-920D-10R-2, the Mg\# values of olivines in the wall spinel harzburgite are constant, near Mg\#=91 (Fig. 6). In the replacement band of spinel harzburgite, the $\mathrm{Mg} \#$ values rapidly decrease from 91 to 72 toward the websterite vein. Such a decreasing trend of $\mathrm{Mg}$ \# toward the magmatic vein can be traced in spinel (Fig. 6) and orthopyroxene (Fig. 7), indicating iron enrichment during the modification. The $\mathrm{NiO}$ content of olivine also decreases from 0.35 to $0.12 \mathrm{wt} \%$ in the replacement band. On the other hand, the Cr\# and the $\mathrm{TiO}_{2}$ content of spinel (Fig. 6), and $\mathrm{FeO}^{*}$ and $\mathrm{TiO}_{2}$ contents of orthopyroxene and clinopyroxene (Fig. 7) increase in the replacement bands. No depletions in melt components can be detected in the replacement bands.

In Piece 7 of Section 153-920D-13R-2, a notable depletion in $\mathrm{Mg} \#$ values of olivine and spinel and enrichments in $\mathrm{FeO}^{*}$ and $\mathrm{TiO}_{2}$ of spinel, orthopyroxene, and clinopyroxene are observed (Fig. 8). The across-channel variations that occurred during modification in this piece are also characterized by enrichments in $\mathrm{FeO}^{*}$ and $\mathrm{TiO}_{2}$ in the spinel harzburgite replacement bands.

The websterite vein crosscutting the spinel harzburgite (Piece 4 of 153-920D-10R-2) contains extremely FeO*-rich and NiO-poor olivines $(\mathrm{Mg} \#=72, \mathrm{NiO}=0.1-0.2 \mathrm{wt} \%)$, ilmenites, and $\mathrm{FeO}^{*}$, $\mathrm{TiO}_{2}$-rich and $\mathrm{MgO}-, \mathrm{Al}_{2} \mathrm{O}_{3^{-}}$, and $\mathrm{Cr}_{2} \mathrm{O}_{3}$-poor orthopyroxenes and clinopyroxenes (Figs. 6, 7). The websterite vein in Piece 7 of 153-920D-13R2 is also characterized by the mineralogy rich in $\mathrm{FeO}^{*}$ and $\mathrm{TiO}_{2}$, and poor in $\mathrm{MgO}, \mathrm{Al}_{2} \mathrm{O}_{3}, \mathrm{NiO}$, and $\mathrm{Cr}_{2} \mathrm{O}_{3}$ (Fig. 8).

\section{DISCUSSION}

\section{Characteristics of Chemical Composition of Primary Minerals}

The MARK harzburgite and lherzolite from Hole 920D contain primary minerals with uniform compositions (Figs. 1-5), although there are some deviations from these compositions in the modified harzburgite and lherzolite samples (Figs. 1-3). The Mg\# values of primary olivine $(\mathrm{Mg} \#=91.0)$, spinel $(\mathrm{Mg} \#=70-75)$, and orthopyroxene $(\mathrm{Mg} \#=91.0)$ in the MARK peridotites indicate the moderately depleted nature. The $\mathrm{Cr} \#$ values of primary spinel $(\mathrm{Cr} \#=27-$ 31 ) and the $\mathrm{Al}_{2} \mathrm{O}_{3}$ content of orthopyroxene ( $3.8 \mathrm{wt} \%$ ) also suggest that the peridotites are moderately depleted. This is consistent with the discussion of mineralogical variability for the Mid-Atlantic Ridge peridotites (e.g., Dick et al., 1984; Michael and Bonatti, 1985; Bonatti et al., 1992). It is noted that the spinel compositions around $\mathrm{Cr} \#=29$ are slightly higher than those from Site 670 in the MARK area (Fujii, 1990; Juteau et al., 1990; Komor et al., 1990), and lower than those from Site 395 in the western flank of Mid-Atlantic Ridge in the MARK area (Arai and Fujii, 1979; Sinton, 1979), Site 334 (DSDP Leg 37) in the FAMOUS area (Clarke and Loubat, 1977; Sigurdsson, 1977; Symes et al., 1977), and some fracture zones around Mid-Atlantic Ridge (Prinz et al., 1976; Bonatti et al., 1992; Cannat et al., 1992).

The extremely low $\mathrm{Ti}$ and $\mathrm{Na}$ contents of the Hole 920D clinopyroxenes (Fig. 4) suggest that the Hole 920D peridotites underwent a higher degree of partial melting in comparison with other suboceanic peridotites in the American-Antarctic and Southwest Indian Ridges (Dick, 1989) and those related to the pre-oceanic rifting during the early stages of continental breakup, such as Saint Paul's Rocks 
Figure 6. Across-channel chemical variations of $\mathrm{Mg \#}$ $(100 \mathrm{Mg} /[\mathrm{Mg}+\mathrm{Fe}])$ and $100 \mathrm{NiO}(\mathrm{wt} \%)$ for olivines, and $\mathrm{Mg \#}$ and $100 \mathrm{TiO}_{2}$ (wt\%) for spinels in Sample 153-920D10R-2 (Piece 4). Horizontal axis is distance (mm) perpendicular to the channel. Vertical lines show the border surface of replacement band (modified spinel harzburgite) and websterite (WEB) vein. Sp. H. = spinel harzburgite.
Sp. $\mathbf{H}$

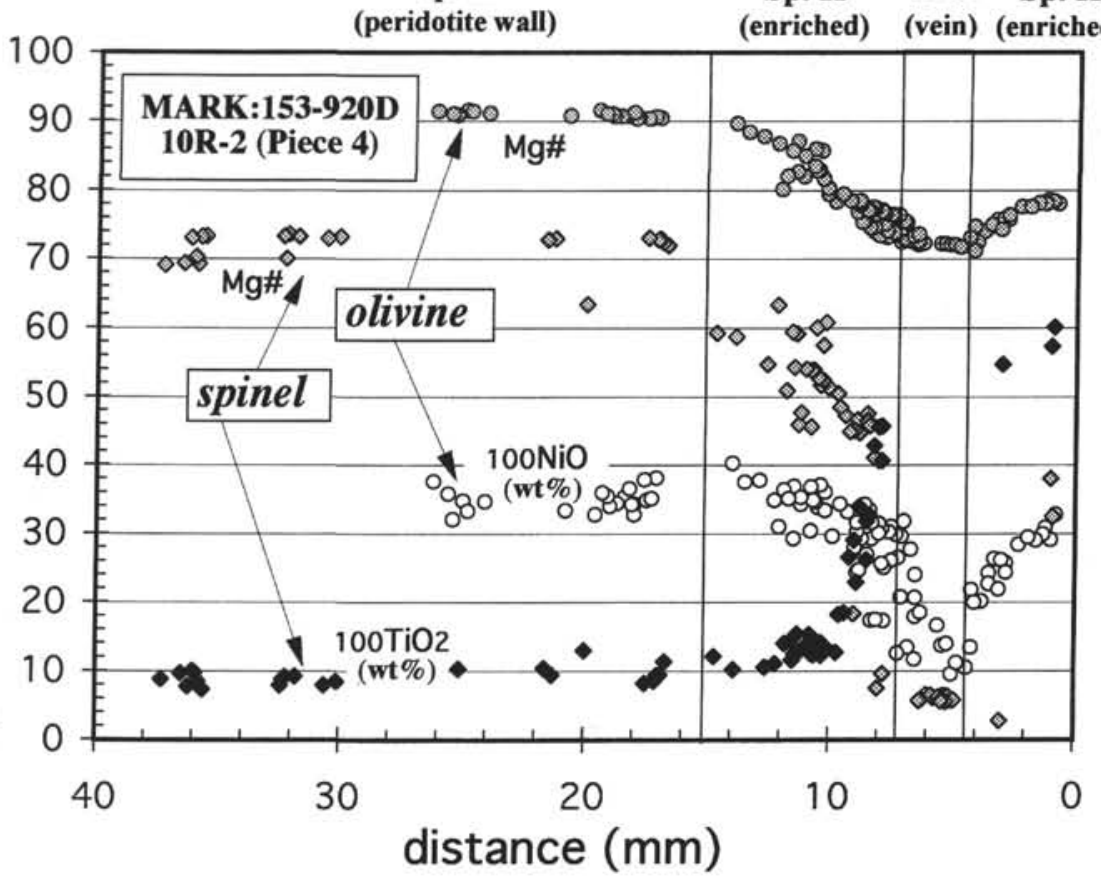

Figure 7. Across-channel chemical variations of $\mathrm{TiO}_{2}$ and $1 / 5 \mathrm{FeO} *$ wt $\%$ for clinopyroxenes and $\mathrm{TiO}_{2}, 1 / 5 \mathrm{FeO} *$, and $1 / 10 \mathrm{MgO} w \mathrm{wt} \%$ for orthopyroxenes in Sample 153-920D10R-2 (Piece 4). Horizontal axis is distance ( $\mathrm{mm}$ ) perpendicular to the channel. Vertical lines are the same lithologic borders as in Figure 6. Abbreviations as in Figure 6.

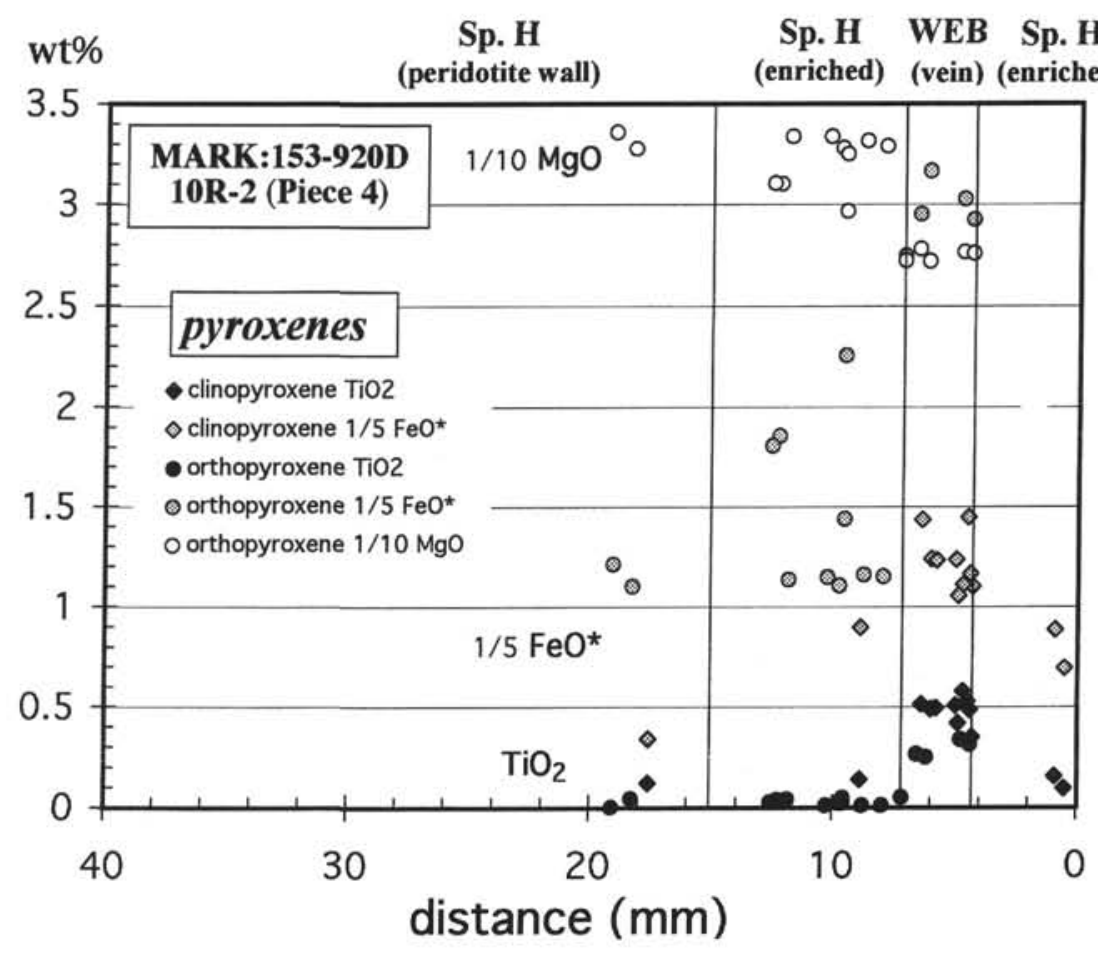

(Melson et al., 1972), Galicia Margin (Evans and Girardeau, 1988; Kornprobst and Tabit, 1988), Zabargad (Bonatti et al., 1986; Piccardo et al., 1988), southwest Australia (Nicholls et al., 1981), and Antarctic Seamount B (Niida and Yuasa, 1995). More depleted suboceanic peridotites with extremely low $\mathrm{Ti}$ and $\mathrm{Na}$ clinopyroxenes have been recently reported from the Hess Deep (Girardeau and Francheteau, 1993).

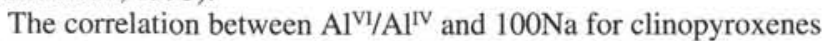
(Fig. 5) is a good indicator of equilibration depths of peridotite as-

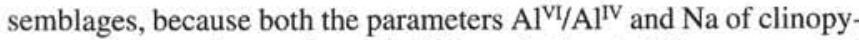
roxenes depend on the solubility of the jadeite component $\left(\mathrm{NaAlSi}_{2} \mathrm{O}_{6}\right.$ ) in pyroxene solid solutions (Aoki and Shiba, 1973). High-pressure clinopyroxenes are expected to be plotted into relatively higher $\mathrm{Al}^{\mathrm{VI}} / \mathrm{Al}^{\mathrm{IV}}$ and $\mathrm{Na}$ areas of Figure 5, although the role of $\mathrm{Na}$ in clinopyroxenes is more complex (e. g., Kornprobst et al., 1981). The notably low ratios of $\mathrm{Al}^{\mathrm{VI}} / \mathrm{Al}^{\mathrm{IV}}(0.403$ to 0.860$)$ and low $100 \mathrm{Na}$ (less than 1.2) for the Hole 920D clinopyroxenes indicate that the peridotites equilibrated at a shallow level in the upper mantle. In- 

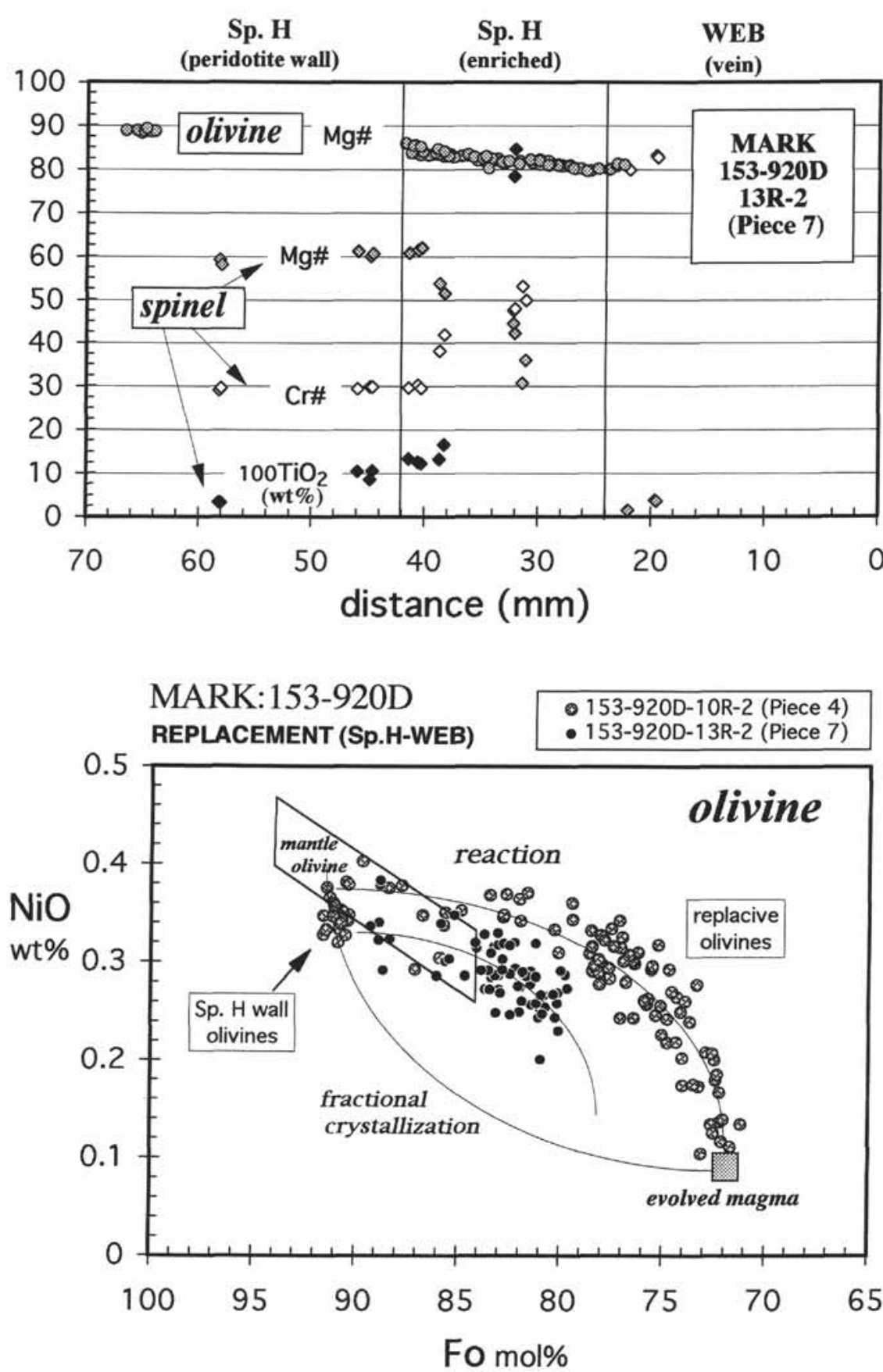

Figure 8. Across-channel chemical variations of $\mathrm{Mg} \#$ $(100 \mathrm{Mg} /[\mathrm{Mg}+\mathrm{Fe}])$ for olivines, and $\mathrm{Mg} \#, \mathrm{Cr} \#(100 \mathrm{Cr} \# /$ $[\mathrm{Cr}+\mathrm{Al}])$, and $100 \mathrm{TiO}_{2}(\mathrm{wt} \%)$ for spinels in Sample 153-920D-13R-2 (Piece 7). Horizontal axis is distance $(\mathrm{mm})$ perpendicular to the channel. Vertical lines show border surface of replacement band (modified spinel harzburgite) and websterite vein. Abbreviations as in Figure 6 .
Figure 9. Plots of $\mathrm{NiO}$ (wt\%) vs. Fo content (mol\%) for olivines in Samples 153-920D-10R-2 (Piece 4) and 13R-2 (Piece 7). Note the wide compositional variations between olivines in spinel harzburgite wall ( $\mathrm{Fo}=91.0 \mathrm{wt} \%$ and $\mathrm{NiO}=0.37 \mathrm{wt} \%)$ and those in evolved magma $(\mathrm{Fo}=74.0$ $\mathrm{wt} \%$ and $\mathrm{NiO}=0.10 \mathrm{wt} \%$ ). This suggests that the modifi5 cation occurred as an open-system reaction between the wall harzburgite and the infiltration melt with highly evolved composition. Abbreviations as in Figure 6. terstitial clinopyroxenes with magmatic twins reported from the MARK peridotites (Cannat, Karson, Miller, et al., 1995) support the estimation of a "shallow-level" equilibration depth.

\section{Modification in a Cold Peridotite Wall}

Mineralogical heterogeneity caused by variable degrees of partial melting in upper mantle has been discussed for peridotites from the Mid-Atlantic Ridge area (e.g., Dick et al., 1984; Michael and Bonatti, 1985; Bonatti et al., 1992). Melt extraction from the upwelling mantle beneath spreading ridges appears to be a major process that can produce a wide compositional range of suboceanic peridotites. Local melt extraction, generated within a shallow-level mantle by magma channeling, also produces highly depleted peridotites (e.g., Dick,
1977; Quick, 1981). In addition, melt impregnation and melt/rock interaction appear to occur as pervasive processes in mantle peridotite (Kelemen et al., 1992, 1995; Boudier and Nicolas, 1995). Recent discussions about impregnated peridotites from mid-ocean ridge areas suggest that melt impregnation may enrich wall peridotites in melt components (Cannat et al., 1990; Girardeau and Mercier, 1992; Girardeau and Francheteau, 1993; Hekinian et al., 1993).

Strongly modified peridotites, recovered from Hole 920D, are mineralogically enriched in $\mathrm{FeO}^{*}$ and $\mathrm{TiO}_{2}$. As examined in Samples 153-920D-10R-2 (Piece 4) and 153-920D-13R-2 (Piece 7), the enrichment generated by magma channeling was restricted to narrow replacement bands of modified spinel harzburgites in contact with websterite veins (Figs. 6-8). It is expected from the extremely FeO*and $\mathrm{TiO}_{2}$-rich mineralogy of the websterite veins that the agents of 
modification were silicate melts, rich in $\mathrm{FeO}^{*}$ and $\mathrm{TiO}_{2}$, that have an evolved ferrogabbroic composition infiltrated from the veins. On the other hand, the unmodified harzburgite wall is characterized by "uniform" mineral compositions. No evidence for melt extraction from the harzburgite wall during magma channeling has been detected in Hole 920D. This pattern indicates that the replacement process occurred as a one-way addition of melts from the channeling magma and that the modification occurred in a "cold" harzburgite wall whose temperature was below the solidus.

Figure 9 presents a further analysis of olivine compositions in the modified harzburgites crosscut by the websterite veins. The modified olivines in both harzburgite pieces examined have wide compositional ranges in terms of Fo-NiO correlation. In particular, the Fo content of olivines in Piece 4 of 153-920D-10R-2 varies from 91.0 to 72.0 , and their $\mathrm{NiO}$ wt $\%$ varies from 0.37 to 0.09 . The variation trend fully connects the primary compositions ( $\mathrm{Fo}=91.0, \mathrm{NiO}=0.37 \mathrm{wt} \%$ ) defined by olivines from the spinel harzburgite wall and those (Fo = $72.0, \mathrm{NiO}=0.09 \mathrm{wt} \%$ ) from the evolved magma. The trend is definitely separate from the olivine fractionation line expected. The olivines close to the unmodified wall harzburgite show a slight depletion in $\mathrm{NiO}$ with the $\mathrm{FeO}$ * enrichment, whereas the olivines close to the websterite vein show a rapid depletion in $\mathrm{NiO}$. This variation is well explained to have been formed by melt-olivine reaction, which may produce a slight depletion of $\mathrm{NiO}$ if the reacted melt/solid mass ratios are rather small (Barnes, 1986). It is noted that an open-system reaction between melt and olivine is the unique explanation for such a slight $\mathrm{NiO}$ depletion with strong $\mathrm{FeO}$ * enrichment of olivine (Ozawa, 1994).

\section{SUMMARY}

Serpentinized peridotites drilled at Site 920 in the MARK area are 95\% spinel harzburgite with small amounts of spinel lherzolite and spinel dunite. The peridotites recovered from Hole 920D are crosscut by several magmatic dikes, veins, and veinlets, which are olivine gabbro, gabbro, oxide-rich gabbro, pyroxenite, oxide-rich pyroxenite, and plagioclase-olivine phyric diabase.

The primary mineralogies of the Hole 920D harzburgites and lherzolites are characterized by uniform compositions of primary olivine $(\mathrm{Mg} \#=91.0, \mathrm{NiO}=0.37 \mathrm{wt} \%)$, spinel $(\mathrm{Mg} \#=70-75, \mathrm{Cr} \#=27-$ $31)$, orthopyroxene $\left(\mathrm{Mg} \#=91.0, \mathrm{Al}_{2} \mathrm{O}_{3}=3.8 \mathrm{wt} \%\right)$, and clinopyroxene $\left(\mathrm{TiO}_{2}<0.15 \mathrm{wt} \%, \mathrm{Na}_{2} \mathrm{O}<0.11 \mathrm{wt} \%\right)$, suggesting the moderately depleted peridotite signature.

Modification from the primary mineralogy can be detected as enrichments in $\mathrm{FeO}^{*}$ and $\mathrm{TiO}_{2}$, and depletions in $\mathrm{MgO}, \mathrm{NiO}$, and $\mathrm{Al}_{2} \mathrm{O}_{3}$ in limited peridotite samples next to the magmatic dikes, veins, and veinlets. A model of one-way melt addition from the channeling magma and open-system reaction between the wall harzburgite and the infiltration melts with a highly evolved composition was discussed, based on examinations of variations in across-channel chemical composition for major constituent minerals. The modification event is considered to have occurred as final magma channeling in "cold" peridotites at a shallow level of the upper mantle.

\section{ACKNOWLEDGMENTS}

The author is grateful to the scientists, technicians, officers, and crews aboard the JOIDES Resolution, and the shore-based ODP staff for their support and cooperation during and after Leg 153. Thanks are also for technical assistance by S. Terada, T. Kuwajima, and $\mathrm{H}$. Nomura of Hokkaido University. Thorough and thoughtful reviews by S.C. Komor, B. Wathen, and D. Elthon are greatly appreciated, and the manuscript also benefited from the comments of an anonymous reviewer.

\section{REFERENCES}

Aoki, K., and Shiba, I., 1973. Pyroxenes from lherzolite inclusions of Itinome-gata, Japan. Lithos, 6:41-51.

Arai, S., and Fujii, T., 1979. Petrology of ultramafic rocks from Site 395. In Melson, W.G., Rabinowitz, P.D., et al., Init. Repts. DSDP, 45: Washington (U.S. Govt. Printing Office), 587-594.

Arai, S., and Matsukage, K., 1996. Petrology of the gabbro-troctolite-peridotite complex from Hess Deep, equatorial Pacific: implications for mantle-melt interaction within the oceanic lithosphere. In Mével, C., Gillis, K.M., Allan, J.F., and Meyer, P.S. (Eds.), Proc. ODP, Init. Repts., 147: College Station, TX (Ocean Drilling Program), 135-155.

Barnes, S.J., 1986. The effect of trapped liquid crystallization on cumulus mineral compositions in layered intrusions. Contrib. Mineral. Petrol., 93:524-531.

Bodinier, J.L., Vasseur, G., Vernieres, J., Dupuy, C., and Fabries, J., 1990. Mechanisms of mantle metasomatism: geochemical evidence from the Lherz Orogenic Peridotite. J. Petrol., 31:597-628.

Bonatti, E., and Honnorez, J., 1976. Sections of the earth's crust in equatorial Atlantic. J. Geophys. Res., 81:4104-4116.

Bonatti, E., and Michael, P.J., 1989. Mantle peridotites from continental rifts to ocean basins to subduction zones. Earth Planet. Sci. Lett., 91:297-311.

Bonatti, E., Ottonello, G., and Hamlyn, P.R., 1986. Peridotites from the island of Zabargad (St. John), Red Sea: petrology and geochemistry. $J$. Geophys. Res., 91:599-631.

Bonatti, E., Peyve, A., Kepezhinskas, P., Kurentsova, N., Seyler, M., Skolotnev, S., and Udintsev, G., 1992. Upper mantle heterogeneity below the Mid-Atlantic Ridge, $0^{\circ}-15^{\circ}$ N. J. Geophys. Res., 97:44614476.

Boudier, F., and Nicolas, A., 1995. Nature of the Moho Transition Zone in the Oman Ophiolite. J. Petrol., 36:777-796.

Cannat, M., Bideau, D., and Bougault, H., 1992. Serpentinized peridotites and gabbros in the Mid-Atlantic Ridge axial valley at $15^{\circ} 37^{\prime} \mathrm{N}$ and $16^{\circ} 52$ N. Earth Planet. Sci. Lett., 109:87-106.

Cannat, M., Bideau, D., and Hébert, R., 1990. Plastic deformation and magmatic impregnation in serpentinized ultramafic rocks from the Garrett transform fault (East Pacific Rise). Earth Planet. Sci. Lett., 101:216-232.

Cannat, M., Karson, J.A., Miller, D.J., et al., 1995. Proc. ODP, Init. Repts., 153: College Station, TX (Ocean Drilling Program).

Clarke, D.B., and Loubat, H., 1977. Mineral analyses from the peridotitegabbro-basalt complex at Site 334, DSDP Leg 37. In Aumento, F., Melson, W.G., et al., Init. Repts. DSDP, 37: Washington (U.S. Govt. Printing Office), 847-855.

Dick, H.J.B., 1977. Partial melting in the Josephine peridotite, I. The effect of mineral composition and its consequence for geobarometry and geothermometry. Am. J. Sci., 277:801-832.

, 1989. Abyssal peridotites, very slow spreading ridges and ocean ridge magmatism. In Saunders, A.D., and Norry, M.J. (Eds.), Magmatism in the Ocean Basins. Geol. Soc. Spec. Publ. London, 42:71-105.

Dick, H.J.B., and Bullen, T., 1984. Chromian spinel as a petrogenetic indicator in abyssal and alpine-type peridotites and spatially associated lavas. Contrib. Mineral. Petrol., 86:54-76.

Dick, H.J.B., Fisher, R.L., and Bryan, W.B., 1984. Mineralogic variability of the uppermost mantle along mid-ocean ridges. Earth Planet. Sci. Lett., 69:88-106.

Evans, C.A., and Girardeau, J., 1988. Galicia Margin peridotites: undepleted abyssal peridotites from the North Atlantic. In Boillot, G., Winterer, E.L., et al., Proc. ODP, Sci. Results, 103: College Station, TX (Ocean Drilling Program), 195-207.

Frey, F.A., and Green, D.H., 1974. The mineralogy, geochemistry and origin of Lherzolitic inclusions in Victorian basanites. Geochim. Cosmochim. Acta., 38:1023-1059.

Fujii, T., 1990. Petrology of peridotites from Hole 670A, Leg 109. In Detrick, R., Honnorez, J., Bryan, W.B., Juteau, T., et al., Proc. ODP, Sci. Results, 107: College Station, TX (Ocean Drilling Program), 19-25.

Girardeau, J., and Francheteau, J., 1993. Plagioclase-wehrlites and peridotites on the East Pacific Rise (Hess Deep) and the Mid-Atlantic Ridge (DSDP Site 339) evidence for magma percolation in the oceanic upper mantle. Earth Planet. Sci. Lett., 115:137-149.

Girardeau, J., and Mercier, J.-C.C., 1992. Evidence for plagioclase-lherzolite intrusion in the Mid-Atlantic Ridge, DSDP Leg 37. In Parson, L.M., Murton, B.J., and Browning P. (Eds.), Ophiolites and their Modern Oceanic Analogues. Geol. Soc. Spec. Publ. London, 60:241-250. 
Hébert, R., Adamson, A.C., and Komor, S.C., 1990. Metamorphic petrology of ODP Leg 109, Hole 670A serpentinized peridotites: serpentinization processes at a slow spreading ridge environment. In Detrick, R., Honnorez, J., Bryan, W.B., Juteau, T., et al., Proc. ODP, Sci. Results, 106/ 109: College Station, TX (Ocean Drilling Program), 103-115.

Hekinian, R., Bideau, D., Francheteau, J., Cheminée, J.L., Armijo, R., Lonsdale, P., and Blum, N., 1993. Petrology of the East Pacific Rise crust and upper mantle exposed in the Hess Deep (eastern equatorial Pacific). J. Geophys. Res., 98:8069-8094.

Irving, A.J., 1980. Petrology and geochemistry of composite ultramafic xenoliths in alkalic basalts and implications for magmatic processes within the mantle. Am. J. Sci., 280-A:389-426.

Juteau, T., Berger, E., and Cannat, M., 1990. Serpentinized, residual mantle peridotites from the M.A.R. median valley, ODP Hole $670 \mathrm{~A}\left(21^{\circ} 10 \mathrm{~N}\right.$, $45^{\circ} 02 \mathrm{~W}$, Leg 109): primary mineralogy and geothermometry. In Detrick, R., Honnorez, J., Bryan, W.B., Juteau, T., et al., Proc. ODP, Sci. Results, 106/109: College Station, TX (Ocean Drilling Program), 27-45.

Karson, J.A., Thompson, G., Humphris, S.E., Edmond, J.M., Bryan, W.B., Brown, J.R., Winters, A.T., Pockalny, R.A., Casey, J.F., Campbell, A.C., Klinkhammer, G., Palmer, M.R., Kinzler, R.J., and Sulanowska, M.M., 1987. Along-axis variations in seafloor spreading in the MARK area. Nature, 328:681-685.

Keleman, P.B., Dick, H.J.B., and Quick, J.E., 1992. Formation of harzburgite by pervasive melt/rock reaction in the upper mantle. Nature, 358:635641 .

Kelemen, P.B., Shimizu, N., and Salters, V.J.M., 1995. Extraction of midocean-ridge basalt from the upwelling mantle by focused flow of melt in dunite channels. Nature, 375:747-753.

Kempton, P.D., 1987. Mineralogic and geochemical evidence for differing styles of metasomatism in spinel lherzolite xenoliths: enriched mantle source regions of basalts. In Menzies, M.A., and Hawkesworth, C.J. (Eds.), Mantle Metasomatism: London (Academic Press), 45-89.

Komor, S.C., Grove, T.L., and Hébert, R., 1990. Abyssal peridotites from ODP Hole $670 \mathrm{~A}\left(21^{\circ} 10 \mathrm{~N}, 45^{\circ} 02 \mathrm{~W}\right)$ : residues of mantle melting exposed by non-constructive axial divergence. In Detrick, R., Honnorez, J., Bryan, W.B., Juteau, T., et al., Proc. ODP, Sci. Results, 106/109: College Station, TX (Ocean Drilling Program), 85-101.

Kornprobst, J., Ohnenstetter, D., and Ohnenstetter, M., 1981. Na and Cr contents in clinopyroxenes from peridotites: a possible discriminant between sub-continental and sub-oceanic mantle. Earth Planet. Sci. Lett., 53:241254

Kornprobst, J., and Tabit, A., 1988. Plagioclase-bearing ultramafic tectonites from the Galicia Margin (Leg 103, Site 637): comparison of their origin and evolution with low-pressure ultramafic bodies in Western Europe. In Boillot, G., Winterer, E.L., et al., Proc. ODP, Sci. Results, 103: College Station, TX (Ocean Drilling Program), 253-269.

Melson, W.G., Hart, S.R., and Thompson, G., 1972. St. Paul's Rocks, equatorial Atlantic: petrogenesis, radiometric ages, and implications on seafloor spreading. Mem.-Geol. Soc. Am., 132:241-272.

Mével, C., Cannat, M., Gente, P., Marion, E., Auzende, J.-M., and Karson, J.A., 1991. Emplacement of deep crustal and mantle rocks on the west median valley wall of the MARK area (MAR $23^{\circ} \mathrm{N}$ ). Tectonophysics, 190:31-53.
Michael, P.J., and Bonatti, E., 1985. Peridotite composition from the North Atlantic: regional and tectonic variations and implications for partial melting. Earth Planet. Sci. Lett., 73:91-104.

Nicholls, I.A., Ferguson, J., Jones, H., Marks, G.P., and Mutter, J.C., 1981. Ultramafic blocks from the ocean floor southwest of Australia. Earth Planet. Sci. Lett., 56:362-374.

Nicolas, A., and Prinzhofer, A., 1983. Cumulative or residual origin for the transition zone in ophiolites: structural evidence. J. Petrol., 24:188-206.

Nielson, J.E., Budahn, J.R., Unruh, D.M., and Wilshire, H.G., 1993. Actualistic models of mantle metasomatism documented in a composite xenolith from Dish Hill, California. Geochim. Cosmochim. Acta, 57:105-121.

Niida, K., and Yuasa, M., 1995. Peridotites from the seamount off Wilkes Land, Antarctica. Proc. NIPR Symp. Antarct. Geosci., 8:169-180.

Ozawa, K., 1994. Melting and melt segregation in the mantle wedge above a subduction zone: evidence from the chromite-bearing peridotites of the Miyamori Ophiolite Complex, northeastern Japan. J. Petrol., 35:647678.

Piccardo, G.B., Messiga, B., and Vannucci, R., 1988. The Zabargad peridotite-pyroxenite association: petrological constraints on its evolution. Tectonophysics, 150:135-162.

Prinz, M., Keil, K., Green, J.A., Reid, A.M., Bonatti, E., and Honnorez, J., 1976. Ultramafic and mafic dredge samples from the Equatorial MidAtlantic Ridge and fracture zones. J. Geophys. Res., 81:4087-4103.

Quick, J.E., 1981. The origin and significance of large, tabular dunite bodies in the Trinity peridotite, northern California. Contrib. Mineral. Petrol. 78:413-422.

Sigurdsson, H., 1977. Spinels in Leg 37 basalts and peridotites: phase chemistry and zoning. In Aumento, F., Melson, W.G., et al., Init. Repts. DSDP, 37: Washington (U.S. Govt. Printing Office), 883-891.

Sinton, J.M., 1979. Petrology of (alpine-type) peridotites from Site 395, DSDP Leg 45. In Melson, W.G., Rabinowitz, P.D., et al., Init. Repts. DSDP, 45: Washington (U.S. Govt. Printing Office), 595-601.

Streckeisen, A., 1973. Plutonic rocks: classification and nomenclature recommended by the IUGS Subcommission on the Systematics of Igneous Rocks. Geotimes, 18:26-30.

Symes, R.F., Bevan, J.C., and Hutchinson, R., 1977. Phase chemistry studies on gabbro and peridotite rocks from Site 334, DSDP Leg 37. In Aumento, F., Melson, W.G., et al., Init. Repts. DSDP, 37: Washington (U.S. Govt. Printing Office), 841-844.

Takahashi, E., Uto, K., and Schilling, J.-G., 1987. Primary magma compositions and $\mathrm{Mg} / \mathrm{Fe}$ ratios of their mantle residues along Mid Atlantic Ridge $29^{\circ} \mathrm{N}$ to $73^{\circ} \mathrm{N}$. Tech. Rep. ISEI, Okayama Univ., Ser. A, 9:1-14.

Takazawa, E., Frey, F.A., Shimizu, N., Obata, M., and Bodinier, J.L., 1992. Geochemical evidence for melt migration and reaction in the upper mantle. Nature, 359:55-58.

Wilshire, H.G., and Shervais, J.W., 1975. Al-augite and Cr-diopside ultramafic xenoliths in basaltic rocks from western United States. Phys. Chem. Earth, 9:257-272.

Date of initial receipt: 7 August 1995

Date of acceptance: 14 December 1995

Ms 153SR-014 